\title{
Fuzzy mathematical models for the analysis of fuzzy systems with application to liver disorders
}

\author{
Rana Waleed Hndoosh ${ }^{1}$, Sanjeev Kumar ${ }^{2}$, M. S. Saroa ${ }^{3}$ \\ ${ }^{1}$ Department of Mathematics, Ph.D Student, India. \\ ${ }^{3}$ Dept. of Mathematics, Dr. B. R. Ambedkar University, India. \\ ${ }^{2}$ Dept. of Mathematics, Maharishi Markandeshawar University, India.
}

\begin{abstract}
The main objective of this model is to focus on how to use the model of fuzzy system to solve fuzzy mathematics problems. Some mathematical models based on fuzzy set theory, fuzzy systems and neural network techniques seem very well suited for typical technical problems. We have proposed an extension model of a fuzzy system to N-dimension, using Mamdani's minimum implication, the minimum inference system, and the singleton fuzzifier with the center average defuzzifier. Here construct two different models namely a fuzzy inference system and an adaptive fuzzy system using neural network. We have extended the theorem for accuracy of the fuzzy system to $N$ - dimensions, and provided a medical application of the fuzzy mathematics models. Since, liver is the largest internal member in the human body, so diagnosing liver disorder disease is a high interest to researchers of the fuzzy modeling and the fuzzy system. Therefore, the fuzzy mathematical models are applied on a real data to the Liver Disorder disease. Consequently, a comparison between three models: the FS with Mamdani model, ST model, and the ANFIS is made. Therefore, we have obtained the best result with the ANFIS. Finally, the programs of these models by using MATLAB created and performed.
\end{abstract}

Keywords: Fuzzy system; FIS; ANFIS; Neural networks; Accuracy of the fuzzy system; Liver disorders.

\section{Introduction}

Fuzzy mathematics provides the starting point and basic language for fuzzy systems (FSs) and fuzzy modeling (FM) [Ruan and Wang (1997)], while the fuzzy mathematical principles are developed by replacing the sets in classical mathematical theory with fuzzy sets (FSs) [Samandar (2011)]. The concepts and principles in fuzzy mathematics are useful in FSs and adaptive neuro-fuzzy systems (ANFSs) [Singh et al. (2009)], [Shing and Jang (1993)]. Fuzzy variables are processed using a system called a fuzzy inference system (FIS) which involves fuzzification, fuzzy inference, and defuzzification [Wang (1997)], [Aik and Jayakumar (2008)]. The FIS collects the rules in the fuzzy rule-base into a mapping from fuzzy set $\widehat{A} \in X$ to fuzzy set $\widehat{B} \in Y$ [Sivanandam and Deepa (2008)]. We must construct interfaces that are the fuzzifier and defuzzifier, between the FIS and the environment because in most applications the input and output of the FS are real valued numbers such our application in this model to Liver Disorders [Sug (2012) and Gulia et al. (2014)]. The reason to represent a fuzzy system in terms of a neural network is to utilize the learning ability of neural networks to improve performance, such as adaptation of FS [Rameshkumar and Arumugam (2011)]. When the expert is demonstrating, we measure the inputs and the outputs; that is, we can collect a set of input-output data pairs [Nayak (2004)], [Hndoosh et al. (2012) and (2013)]. Therefore, the knowledge is transformed into a set of inputoutput pairs. The task in this work is to model a FS that describes the input-output behavior represented by the input-output pairs and apply the model to Liver Disorders. We will model the FS by first assigning its structure and then tuning its parameters [Jose et al. (1999)]. To simulate the modeling system, need a mathematical model of the Liver Disorders that is described by linguistic variables and its membership functions (MFs) [Chai et al. (2009)]. We note that the fuzzy modeler can successfully control and handle the real data of any problem. As well as, the prediction accuracy is improved by defining more FSs for each input variable [Marza and Seyyedi (2008)]. The advantage of using the FS is that the parameters of MFs have clear physical meanings and we have models to choose good initial values for them [Doğan et al. (2007). We can recover the fuzzy if-then rules that model the FS [Belohlavek and Klir (2011)]. These recovered fuzzy if-then rules may help to demonstrate the modeled FS in a user-friendly manner. The work is divided into four Sections. Section 1 introduces the fundamental concepts and principles in the general field of fuzzy theory that are particularly useful in FSs and ANFIS [Sivanandam and Deepa (2008)]. In Section 2, we have provided the detailed mathematical formulas of the FIS, and we construct interfaces between the FIS and the environment using fuzzifier and defuzzifier models [Jandaghi et al. (2010)].

In the first part of this Section, we propose and extend the model of the FS, the work of Hndoosh et al. (2013) and Wang (1998), from 2-dimention to $N$-dimension using Mamdani's minimum implication with the minimum inference system, the singleton fuzzifier, and center average defuzzifier [Rojas (1996)]. In the second 
part, we have provided the theorem for accuracy of a proposed model [Hndoosh et al. (2012), (2013), and (2014)]. This approach requires $N$-pieces of information in order to model FS to satisfy any pre-specified degree of accuracy [Kamel and Hassan (2009)]. As well as, we have adapted the structure of the FS and modeled of an adaptive FS using a neural network through the third part of this Section [Singh et al. (2009)], [Shing, and Jang (1993)]. In Section 3, we have applied all the previous concepts and models on a real application to Liver Disorders [Sug (2012) and Gulia et al. (2014)], and we have structured of the applied model at the first part. Second and third subsection, provided discussion and results for the model of the FS with Mamdani and ST models and the adaptive FS using neural network, respectively. Consequently, obtained good results of the models, and created programs for the different models using 'MATLAB'. Concluding remarks are present in Section 4. Finally, Appendix is provided the representation of results of the FIS with the Mamdani and ST models, and the results of the ANFIS with their errors through Table 3.

\section{Proposal Of A New Model Of A Fuzzy System On N-Dimensions}

In this section, proposed a model of a fuzzy system, that is extension of the work of Hndoosh et al. (2013) and Wang (1997), from 2-dimention to N-dimension [Ruan and Wang (1997)]. Consider the general membership function of fuzzy set, $A$, is a continuous function in $\mathrm{R}$ given by:

$\mu(x ; a, b, c, d)=\left\{\begin{array}{cc}0 & \text { if } x<a \\ a(x) & \text { if } a \leq x<b \\ 1 & \text { if } b \leq x \leq c \\ d(x) & \text { if } c<x \leq d \\ 0 & \text { ifd }<x\end{array}\right.$

where $[a, d] \subset R$ and $a \leq b \leq c \leq d, 0 \leq a(x) \leq 1$ a non-decreasing function is $\in[a, b)$ and $0 \leq d(x) \leq 1$ is a non-increasing function $\in(c, d]$.

If fuzzy sets $A^{1}, A^{2}, \ldots, A^{N} \in W \subset R$ then, they are called:

1. Complete on $W$, if there exists $A^{\mathrm{k}}$ such that $\mu_{A^{k}}(x)>0$, for any $x \in W$.

2. Consistent on $W$ if $\mu_{A^{k}}(x)=1$ for some $x \in W$ implies that $\mu_{A^{j}}(x)=0$, for all $k \neq j$.

3. Normal, consistent and complete with general MFs, $\mu_{A^{j}}\left(x ; a_{j}, b_{j}, c_{j}, d_{j}\right)$. If $A^{1}<A^{2}<\cdots<A^{N}$, then $c_{j} \leq a_{j+1}<d_{j} \leq b_{j+1}$, for $j=1,2, \ldots, N-1$.

In the next section, we will mode a particular type for medical application of Liver Disorders that have some properties [Belohlavek and Klir (2011)], and consider N-inputs fuzzy systems. Now the proposed model is as the following:

\subsection{The Proposed Model}

Let $G(x)$ be defined $G(x): X \subset R^{n} \rightarrow R$, that is, a function on the compact set $X=\left[\alpha_{1}, \beta_{1}\right] \times \ldots \times$ $\left[\alpha_{n}, \beta_{n}\right]$ and the analytic formula of $G(x)$ be unknown. Suppose that for any $x \in U$, we can obtain $G(x)$. Now, to model a fuzzy system that approximates $G(x)$ is main task and model of a fuzzy system as follows:

\section{Step 1:}

Define $N_{j}(j=1,2, \ldots, n)$ fuzzy sets $A_{j}^{1}, A_{j}^{2}, \ldots, A_{j}^{N_{j}} \in\left[\alpha_{j}, \beta_{j}\right]$, which are normal, consistent, and complete with triangular MFs $\mu_{A_{j}^{1}}\left(x_{j} ; a_{j}^{1}, b_{j}^{1}, c_{j}^{1}\right), \ldots, \mu_{A_{j} N_{j}}\left(x_{j} ; a_{j}^{N_{j}}, b_{j}^{N_{j}}, c_{j}^{N_{j}}\right)$, and $A_{j}^{1}<A_{j}^{2}<\cdots<A_{j}^{N_{j}} \quad$ with $a_{j}^{1}=b_{j}^{1}=$ $\alpha_{j}$ and $b_{j}^{N_{j}}=c_{j}^{N_{j}}=\beta_{j}$, which,

- $\quad e_{1}^{1}=\alpha_{1}, e_{1}^{N_{1}}=\beta_{1}$, and $e_{1}^{j}=b_{1}^{j}$ for $j=2,3, \ldots, N_{1}-1$,

- $e_{2}^{1}=\alpha_{2}, e_{2}^{N_{2}}=\beta_{2}$, and $e_{1}^{j}=b_{2}^{j}$ for $j=2,3, \ldots, N_{2}-1$,

-

- $\quad e_{n}^{1}=\alpha_{n}, e_{n}^{N_{n}}=\beta_{n}$, and $e_{1}^{j}=b_{1}^{j}$ for $j=2,3, \ldots, N_{n}-1$

Step 2:

Construct $I=N_{1} \times N_{2} \times \ldots \times N_{n}$ fuzzy if-then rules in the following form:

$R_{X}^{j_{1} \ldots j_{n}}:$ IF $x_{1}$ is $A_{1}^{j_{1}}$ and $x_{2}$ is $A_{2}^{j_{2}}$ and $\ldots$ and $x_{n}$ is $A_{n}^{j_{n}}$ Then $y$ is $B^{j_{1} \ldots j_{n}}$,

where $j_{1}=1,2, \ldots, N_{1}, j_{2}=1,2, \ldots, N_{2}, \ldots, j_{n}=1,2, \ldots, N_{n}$, and the center of the fuzzy set $B^{j_{1} \ldots j_{n}}$, denoted by $\bar{y}^{j_{1} \cdots j_{n}}$, is chosen as:

$\bar{y}^{j_{1} \ldots j_{n}}=G\left(e_{1}^{j_{1}}, \ldots, e_{n}^{j_{n}}\right)$

This is the case when (3) depends on the Mamdani fuzzy rule [Chai et al. (2009)], and the antecedent of our model is connected by "and" [Sivanandam and Deepa (2008)], [Marza and Seyyedi (2008)], then the truth-value evaluation is given by: 
$\vartheta_{i}=\tau\left(\mu_{A_{1}^{j_{1} \ldots j_{n}, i}}\left(x_{1}\right), \mu_{A_{2}^{j_{1} \ldots j_{n}, i}}\left(x_{2}\right), \ldots, \mu_{A_{n}^{j_{1} \ldots j_{n}, i}}\left(x_{n}\right)\right)$

Therefore, from $\mu_{\overline{B^{i}}}(y)=t\left(\vartheta_{i}, \mu_{B^{i}}(y)\right), \forall y \in R$, the fuzzy inference produces the fuzzy set of output by:

$\mu_{\overline{B^{j} 1 \ldots j_{n}, i}}(y)=t\left(\vartheta_{i}, \mu_{B^{j_{1} \ldots j_{n}, i}}(y)\right) \forall y \in R$

The consequents of all the rules are aggregated in the consequents by the 'max' function as:

$\mu_{B^{j 1 \ldots j n}}(y)=s\left(\mu_{B^{j_{1} \ldots j_{n}, 1}}(y), \mu_{B^{j_{1} \ldots j_{n}, 2}}(y), \ldots, \mu_{B^{j_{1} \ldots j_{n}, i}}(y)\right)$

However, when the consequent of rule is a linear function, then the output of the Sugeno rule depends on function as follows:

$\bar{y}^{j_{1} \ldots j_{n}}=f_{i}\left(x_{1}, x_{2}, \ldots, x_{n}\right)$,

where $f_{i}$ is linear function base on $x_{j}$, that is defined as:

$f_{i}\left(x_{1}, x_{2}, \ldots, x_{n}\right)=a_{1}^{i} x_{1}+a_{2}^{i} x_{2}+\cdots+a_{n}^{i} x_{n}+a_{n+1}^{i}$,

where $a_{j}^{i}$ are the parameters, and can be computed by the least square model.

\section{Step3:}

Constructing the fuzzy system $f(x)$ from the $N_{1} \times N_{2} \times \ldots \times N_{n}$ rules of (4) using Mamdani's minimum implication (MMI) (10a) with the minimum inference system (MIS) (10b), the Singleton fuzzifier (SF) (11), and the Center Average Defuzzifier (CAD) (12), are as follows:

$\left(\mu_{Q_{I M}}(x, y)=\min \left[\mu_{A_{1}}(x), \quad \mu_{A_{2}}(y)\right], Q_{I M} \in X \times Y\right)$

$\mu_{B^{\prime}}(y)=\max _{\forall i}\left[\sup _{x \in X} \min \left(\mu_{A^{\prime}}(x), \mu_{A_{1}^{i}}\left(x_{1}\right), \ldots, \mu_{A_{n}^{i}}\left(x_{n}\right), \mu_{B^{i}}(y)\right)\right]$

$\mu_{A^{\prime}}(x)= \begin{cases}1 & \text { if } x=x^{*} \\ 0 & \text { otherwice }\end{cases}$

$y^{*}=\frac{\sum_{i=1}^{I} \bar{y}^{i} w_{i}}{\sum_{i=1}^{I} w_{i}}$

Therefore, we obtain:

$f(x)=\frac{\sum_{j_{1}=1}^{N_{1} \ldots} \sum_{j_{n}=1}^{N_{n}} \bar{y}^{j_{1} \ldots j_{n}}\left(\min \left(\mu_{A_{1}^{j_{1}}}\left(x_{1}\right), \ldots, \mu_{A_{n}^{j_{n}}}\left(x_{n}\right)\right)\right)}{\sum_{j_{1}=1}^{N_{1}} \ldots \sum_{j_{n}=1}^{N_{n}}\left(\min \left(\mu_{A_{1}^{j_{1}}}\left(x_{1}\right) \ldots, \mu_{A_{n}^{j_{n}}}\left(x_{n}\right)\right)\right)}$

Since the fuzzy sets $A_{j}^{1}, \ldots, A_{j}^{N_{j}}$ are complete at every $x \in X$, then there exist $j_{1}, j_{2}, \ldots, j_{n}$ such that:

$\min \left(\mu_{A_{1}^{j_{1}}}\left(x_{1}\right), \mu_{A_{2}^{j_{2}}}\left(x_{2}\right), \ldots, \mu_{A_{n}^{j_{n}}}\left(x_{n}\right)\right) \neq 0$.

Consequently, the fuzzy system (13) is well defined. From step 2, we note that the antecedent of the rules (4) constitute all the possible sets of the fuzzy sets defined for each input variable [Hndoosh (2013)]. The total number of rules is $N_{n}$, that increases exponentially with the dimension of the input space [Jandaghi (2010)]. In the second part, we explain the accuracy of the $\mathrm{f}(\mathrm{x})$ modeled above on the unknown function $\mathrm{G}(\mathrm{x})$, is explained [Nayak (2004)], [Kamel and Hassan (2009)]. Here extended the accuracy of the fuzzy system from 2dimention to $\mathrm{N}$-dimention, as well as changed the type of fuzzy inference system to a minimum inference system to suit any application [Wang (1997)].

\subsection{Theorem (The Fuzzy System Accuracy for the Proposed Model)}

Let $f(x)$ be the fuzzy system in (13) and $G(x)$ be the unknown function in (4). If $G(x)$ is continuously differentiable on $X=\left[\alpha_{1}, \beta_{1}\right] \times\left[\alpha_{2}, \beta_{2}\right] \times \ldots \times\left[\alpha_{n}, \beta_{n}\right]$, then:

$\|G-f\|_{\infty} \leq\left\|\frac{\partial G}{\partial x_{1}}\right\|_{\infty} h_{1}+\left\|\frac{\partial G}{\partial x_{2}}\right\|_{\infty} h_{2}+\cdots+\left\|\frac{\partial G}{\partial x_{n}}\right\|_{\infty} h_{n}$,

where the infinite norm $\|.\|_{\infty}$ is defined as: $\|d(x)\|_{\infty}=\sup _{x \in X}|d(x)|$ and $h_{j}=\max _{1 \leq k \leq N_{j}}\left|e_{j}^{k+1}-e_{j}^{k}\right|,(j=$ $1,2, \ldots, n$.

Proof:

Let $X^{j_{1} \ldots j_{n}}=\left[e_{1}^{j_{1}}, e_{1}^{j_{1}+1}\right] \times\left[e_{2}^{j_{2}}, e_{2}^{j_{2}+1}\right] \times \ldots \times\left[e_{n}^{j_{n}}, e_{n}^{j_{n}+1}\right]$, where $j_{1}=1,2, \ldots, N_{1}-1, j_{2}=1,2, \ldots, N_{2}-1, \ldots$, $j_{n}=1,2, \ldots, N_{n}-1$. Since $\left[\alpha_{j}, \beta_{j}\right]=\left[e_{j}^{1}, e_{j}^{2}\right] \cup\left[e_{j}^{2}, e_{j}^{3}\right] \cup \ldots \cup\left[e_{j}^{N_{j}-1}, e_{j}^{N_{j}}\right], j=1,2, \ldots, n$., then:

$X=\left[\alpha_{1}, \beta_{1}\right] \times\left[\alpha_{2}, \beta_{2}\right] \times \ldots \times\left[\alpha_{n}, \beta_{n}\right]=\bigcup_{j_{1}=1}^{N_{1}-1} \bigcup_{j_{2}=1}^{N_{2}-1} \ldots \bigcup_{j_{n}=1}^{N_{n}-1} X^{j_{1} j_{2} \ldots j_{n}}$, 
which implies that for any $x \in X$, there exists $X^{j_{1} \ldots j_{n}}$ such that $x \in X^{j_{1} \ldots j_{n}}$.

Now suppose $x \in X^{j_{1} \ldots j_{n}}$, that is $x_{1} \in\left[e_{1}^{j_{1}}, e_{1}^{j_{1}+1}\right], x_{2} \in\left[e_{2}^{j_{2}}, e_{2}^{j_{2}+1}\right], \ldots, x_{n} \in\left[e_{n}^{j_{n}}, e_{n}^{j_{n}+1}\right]$ (since $x$ fixed, $j_{n}$ are also fixed in the sequel). Since the fuzzy sets $A_{1}^{1}, A_{1}^{2}, \ldots, A_{1}^{N_{1}}$ are normal, consistent, and complete, at least one and at most two, $\mu_{A_{1}^{k_{1}}}\left(x_{1}\right)$ are non-zero for $k_{1}=1, \ldots, N_{1}$. From the definition of $e_{1}^{j_{1}}\left(j_{1}=1,2, \ldots, N_{1}-1\right)$, these two possible non-zero $\mu_{A_{1}^{k_{1}}}\left(x_{1}\right)^{\prime}$ s are $\mu_{A_{1}^{j_{1}}}\left(x_{1}\right)$ and $\mu_{A_{1}^{j_{1}+1}}\left(x_{1}\right)$. Similarly upto, the two possible non-zero $\mu_{A_{n}^{k_{n}}}\left(x_{n}\right)^{\prime} s$ ( for $\left.k_{n}=1, \ldots, N_{n}\right)$ are $\mu_{A_{n}^{j_{n}}}\left(x_{n}\right)$ and $\mu_{A_{n}^{j_{n}+1}}\left(x_{n}\right)$. Hence, the fuzzy system $f(x)$ of (16) is simplified as the following:

$$
f(x)=\frac{\sum_{k_{1}=j_{1}}^{j_{1}+1} \ldots \sum_{k_{n}=j_{n}}^{j_{n}+1} \bar{y}^{k_{1} \ldots k_{n}}\left(\min \left(\mu_{A_{1}^{k_{1}}}\left(x_{1}\right), \mu_{A_{2}^{k_{2}}}\left(x_{2}\right), \ldots, \mu_{A_{n}^{k_{n}}}\left(x_{n}\right)\right)\right)}{\sum_{k_{1}=j_{1}}^{j_{1}+1} \ldots \sum_{k_{n}=j_{n}}^{j_{n}+1} \min \left(\mu_{A_{1}^{k_{1}}}\left(x_{1}\right), \mu_{A_{2}^{k_{2}}}\left(x_{2}\right), \ldots, \mu_{A_{n}^{k_{n}}}\left(x_{n}\right)\right)}
$$

From (4), we obtain:

$$
\begin{aligned}
& f(x)=\sum_{k_{1}=j_{1}}^{j_{1}+1} \ldots \sum_{k_{n}=j_{n}}^{j_{n}+1}\left[\frac{\min \left(\mu_{A_{1}^{k_{1}}}\left(x_{1}\right), \ldots, \mu_{A_{n}^{k_{n}}}\left(x_{n}\right)\right)}{\sum_{k_{1}=j_{1}}^{j_{1}+1} \ldots \sum_{k_{n}=j_{n}}^{j_{n}+1} \min \left(\mu_{A_{1}^{k_{1}}}\left(x_{1}\right), \ldots, \mu_{A_{n}^{k_{n}}}\left(x_{n}\right)\right)}\right] * G\left(e_{1}^{k_{1}}, \ldots, e_{n}^{k_{n}}\right) \\
& \because \sum_{k_{1}=j_{1}}^{j_{1}+1} \ldots \sum_{k_{n}=j_{n}}^{j_{n}+1}\left[\frac{\min \left(\mu_{A_{1}^{k_{1}}}\left(x_{1}\right), \ldots, \mu_{A_{n}^{k_{n}}}\left(x_{n}\right)\right)}{\sum_{k_{1}=j_{1}}^{j_{1}+1} \ldots \sum_{k_{n}=j_{n}}^{j_{n}+1} \min \left(\mu_{A_{1}^{k_{1}}}\left(x_{1}\right), \ldots, \mu_{A_{n}^{k_{n}}}\left(x_{n}\right)\right)}\right]=1
\end{aligned}
$$

we have:

$$
\begin{aligned}
|G(x)-f(x)| & \leq \sum_{k_{1}=j_{1}}^{j_{1}+1} \ldots \sum_{k_{n}=j_{n}}^{j_{n}+1}\left[\frac{\min \left(\mu_{A_{1}^{k_{1}}}\left(x_{1}\right), \ldots, \mu_{A_{n}^{k_{n}}}\left(x_{n}\right)\right)}{\sum_{k_{1}=j_{1}}^{j_{1}+1} \ldots \sum_{k_{n}=j_{n}}^{j_{n}+1} \min \left(\mu_{A_{1}^{k_{1}}}\left(x_{1}\right), \ldots, \mu_{A_{n}^{k_{n}}}\left(x_{n}\right)\right)}\right] *\left|G(x)-G\left(e_{1}^{k_{1}}, \ldots, e_{n}^{k_{n}}\right)\right| \\
& \leq \max _{\substack{k_{1}=j_{1}: j_{1}+1 \\
k_{n}=j_{n}: j_{n}+1}}\left|G(x)-G\left(e_{1}^{k_{1}}, \ldots, e_{n}^{k_{n}}\right)\right|
\end{aligned}
$$

From the Mean Value model, may be written (20) as:

$$
|G(x)-f(x)| \leq \max _{\substack{k_{1}=j_{1}, j_{1}+1 \\ k_{n}=j_{n}, j_{n}+1}}\left(\left\|\frac{\partial G}{\partial x_{1}}\right\|_{\infty}\left|x_{1}-e_{1}^{k_{1}}\right|+\left\|\frac{\partial G}{\partial x_{2}}\right\|_{\infty}\left|x_{2}-e_{2}^{k_{2}}\right|+\cdots+\left\|\frac{\partial G}{\partial x_{n}}\right\|_{\infty}\left|x_{n}-e_{n}^{k_{n}}\right|\right)
$$

Since $x \in X^{j_{1} \ldots j_{n}}$, means that $x_{1} \in\left[e_{1}^{j_{1}}, e_{1}^{j_{1}+1}\right], x_{2} \in\left[e_{2}^{j_{2}}, e_{2}^{j_{2}+1}\right] \ldots x_{n} \in\left[e_{n}^{j_{n}}, e_{n}^{j_{n}+1}\right]$, we have, $\left|x_{1}-e_{1}^{k_{1}}\right| \leq\left|e_{1}^{j_{1}+1}-e_{1}^{j_{1}}\right|,\left|x_{2}-e_{2}^{k_{2}}\right| \leq\left|e_{2}^{j_{2}+1}-e_{2}^{j_{2}}\right| \ldots$, and $\left|x_{n}-e_{n}^{k_{n}}\right| \leq\left|e_{n}^{j_{n}+1}-e_{n}^{j_{n}}\right|$ for $k_{1}=j_{1}, j_{1}+$ $1, k_{2}=j_{2}, j_{2}+1, \ldots$, and $k_{n}=j_{n}, j_{n}+1$

Then (21) becomes:

$$
|G(x)-f(x)| \leq\left\|\frac{\partial G}{\partial x_{1}}\right\|_{\infty}\left|e_{1}^{j_{1}+1}-e_{1}^{j_{1}}\right|+\left\|\frac{\partial G}{\partial x_{2}}\right\|_{\infty}\left|e_{2}^{j_{2}+1}-e_{2}^{j_{2}}\right|+\cdots+\left\|\frac{\partial G}{\partial x_{n}}\right\|_{\infty}\left|e_{n}^{j_{n}+1}-e_{n}^{j_{n}}\right|
$$

Since $\|d(x)\|_{\infty}=\sup _{x \in X}|d(x)|$ then $\|G-f\|_{\infty}=\sup _{x \in X}|G-f|$, we get:

$\|G-f\|_{\infty} \leq\left\|\frac{\partial G}{\partial x_{1}}\right\|_{\infty}^{x \in X} \max _{1 \leq j_{1} \leq N_{1}-1}\left|e_{1}^{j_{1}+1}-e_{1}^{j_{1}}\right|+\cdots+\left\|\frac{\partial G}{\partial x_{n}}\right\|_{\infty} \max _{1 \leq j_{n} \leq N_{n}-1}\left|e_{n}^{j_{n}+1}-e_{n}^{j_{n}}\right|$

$\therefore\|G-f\|_{\infty} \leq\left\|\frac{\partial G}{\partial x_{1}}\right\|_{\infty} h_{1}+\left\|\frac{\partial G}{\partial x_{2}}\right\|_{\infty} h_{2}+\cdots+\left\|\frac{\partial G}{\partial x_{n}}\right\|_{\infty} h_{n}$

From (22), we can conclude that fuzzy systems in the form of (17).

Specifically, since $\left\|\frac{\partial G}{\partial x_{1}}\right\|,\left\|\frac{\partial G}{\partial x_{2}}\right\|, \ldots,\left\|\frac{\partial G}{\partial x_{n}}\right\|$ are finite numbers for any given $\varepsilon>0$, we can choose $h_{1}, h_{2}, \ldots, h_{n}$ small enough such that $\left\|\frac{\partial G}{\partial x_{1}}\right\|_{\infty} h_{1}+\left\|\frac{\partial G}{\partial x_{2}}\right\|_{\infty} h_{2}+\cdots+\left\|\frac{\partial G}{\partial x_{n}}\right\|_{\infty} h_{n}<\varepsilon$. Hence from (15) we have:

$\sup _{x \in X}|G-f|=\|G-f\|_{\infty}<\varepsilon$

From (23), we can show that, in order to model a fuzzy system with a pre-specified accuracy, we must know the bounds of the derivatives of $G(x)$ with respect to $x_{1}, x_{2}, \ldots, x_{n}$, that is $\left\|\frac{\partial G}{\partial x_{1}}\right\|_{\infty},\left\|\frac{\partial G}{\partial x_{2}}\right\|_{\infty}, \ldots,\left\|\frac{\partial G}{\partial x_{n}}\right\|_{\infty}$. In the model process, we need to know the value of $G(x)$ at $x$, where $x=\left(e_{1}^{j_{1}}, e_{2}^{j_{2}}, \ldots, e_{n}^{j_{n}}\right)$ for $j_{1}=1,2, \ldots, N_{1}, \quad j_{2}=1,2, \ldots, N_{2}, \ldots, j_{n}=1,2, \ldots, N_{n}$. 
Therefore, this approach requires these $N$ pieces of information in order for the model fuzzy system, to satisfy any pre-specified degree of accuracy.

\subsection{Model of an Adaptive Fuzzy System Using a Neural Network}

In this section, we adapt the structure of the fuzzy system that is specified with the structure of some parameters [Singh et al. (2009)], [Shing and Jang (1993)]. We specify the structure of the fuzzy system to be modeled. Here, we choose the fuzzy system with a MIS, a SF, a CAD, and a Triangular MF [Samandar (2011)], then, we obtain:

$$
f(x)=\frac{\sum_{i=1}^{I} \bar{y}^{i}\left(\min _{\forall j} \mu_{A_{j}^{i}}\left(x_{j}\right)\right)}{\sum_{i=1}^{I}\left(\min _{\forall j} \mu_{A_{j}^{i}}\left(x_{j}\right)\right)}=\frac{\sum_{i=1}^{I} \bar{y}^{i}\left[\min _{\forall j}\left(\max \left(\min _{\forall j}\left(\frac{x_{j}-a_{j}^{i}}{b_{j}^{i}-a_{j}^{i}}, \frac{c_{j}^{i}-x_{j}}{c_{j}^{i}-b_{j}^{i}}, 0\right)\right)\right]\right.}{\sum_{i=1}^{I}\left[\min _{\forall j}\left(\max \left(\min _{\forall j}\left(\frac{x_{j}-a_{j}^{i}}{b_{j}^{i}-a_{j}^{i}}, \frac{c_{j}^{i}-x_{j}}{c_{j}^{i}-b_{j}^{i}}\right), 0\right)\right)\right]}
$$

where $I$ is fixed, and $\bar{y}^{i}, a_{j}^{i}, b_{j}^{i}, c_{j}^{i}$ are free parameters. The fuzzy system (26) has not been modeled because the parameters $\overline{\mathrm{y}}^{\mathrm{i}}, \mathrm{a}_{\mathrm{j}}^{\mathrm{i}}, \mathrm{b}_{\mathrm{j}}^{\mathrm{i}}, \mathrm{c}_{\mathrm{j}}^{\mathrm{i}}$ are not specified [Wang (1997)]. In order to determine these parameters in some optimal manner, it is helpful to represent the fuzzy system $f(x)$ of (26) as a feed-forward network [Jose et al. (1999)]. Specifically, the mapping from the input $x \in U \subset R^{n}$ to the output $f(x) \in V \subset R$ can be performed according to operations [Doğan et al. (2007)]. Note that, the input $x$ is passed through a minimum triangular operator to become:

$z^{i}=\max \left(\min _{\forall j}\left(\frac{x_{j}-a_{j}^{i}}{b_{j}^{i}-a_{j}^{i}}, \frac{c_{j}^{i}-x_{j}}{c_{j}^{i}-b_{j}^{i}}\right), 0\right)$, where $\mathrm{z}^{\mathrm{i}}$ are passed through a summation operator.

Let $K=\sum_{i=1}^{I} z^{i}$ and $L=\sum_{i=1}^{I} \bar{y}^{i} z^{i}$; therefore, the output of the fuzzy system is computed as $f(x)=L / K$. Consequently, we summarize the procedures to model a fuzzy system that depends on layers of network as the following:

\section{Step1: Structure specification and initial parameters}

Select the fuzzy system (26) and determine the number of rule [Rameshkumar and Arumugam (2011)].

The larger number of rule, results more parameters and more computation, but gives better accuracy. Specify the initial parameters $\bar{y}^{i}(0), a_{j}^{i}(0), b_{j}^{i}(0), c_{j}^{i}(0)$, then the initial fuzzy system becomes as in (27). These initial para-meters may be determined according to the linguistic rules from human experts as in our application.

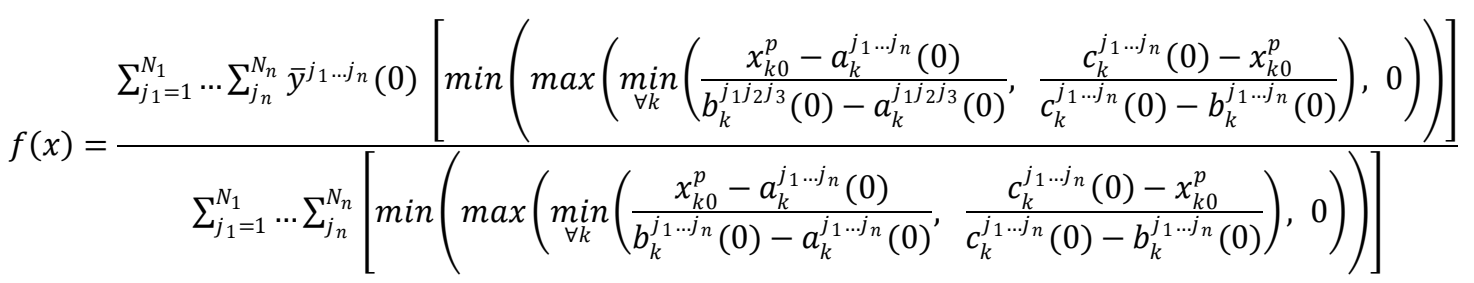

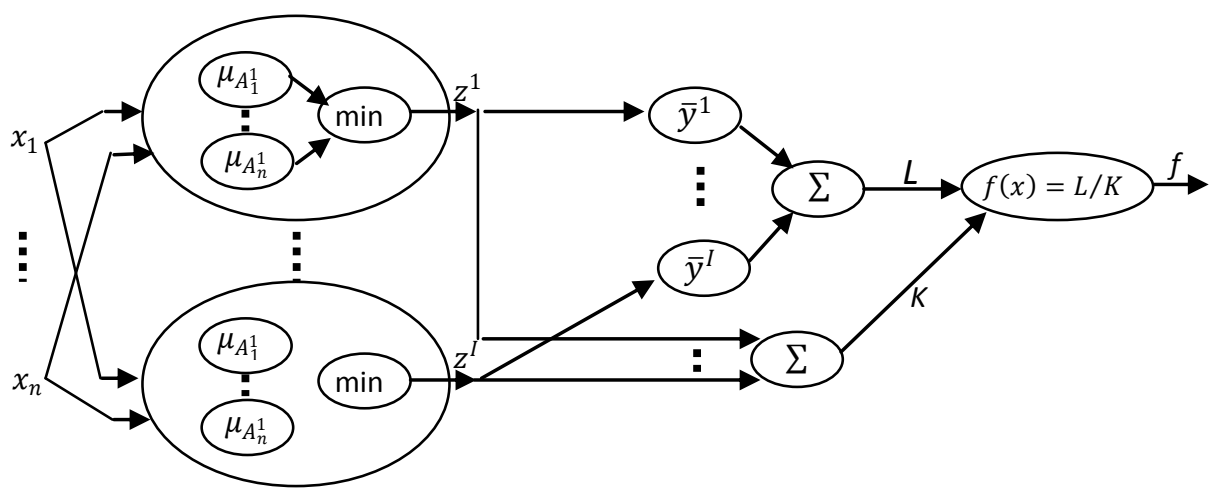

Figure 1: Network representation of the fuzzy system 


\section{Step2: Calculating the outputs of the fuzzy system}

For a given inputs-output pair $\left(x_{k 0}^{p} ; y_{0}^{p}\right), p=1,2, \ldots, k=1,2, \ldots, n$ and at the $q^{\text {th }}$ training stage, $q=0,1, \ldots$, present $x_{k 0}^{p}$ to the input layer of the fuzzy system in Figure 1 and compute the outputs of layers, and therefore, we compute:

First output: Every node produces MF of an input parameter. The node output $o_{1}^{j_{i}}$ is explained by:

$o_{1}^{j_{1}}=\mu_{A_{1}^{j_{1}}}\left(x_{1}\right) ; o_{1}^{j_{2}}=\mu_{A_{2}^{j_{2}}}\left(x_{2}\right), \ldots$, and $o_{1}^{j_{n}}=\mu_{A_{n}^{j_{n}}}\left(x_{n}\right)$,

where $x_{1}, x_{2}, \ldots, x_{n}$ are the inputs, $\mu_{A_{1}^{j_{1}}}, \mu_{A_{2}^{j_{2}}}, \ldots, \mu_{A_{n}^{j_{n}}}$ are linguistic fuzzy sets related with nodes, and $o_{1}^{j_{i}}$ is the degree of MFs of a fuzzy set.

Second output: Every node is a fixed node, whose output is the minimum of all MFs:

$o_{2}^{j_{1} \ldots j_{n}}=z^{j_{1} \ldots j_{n}}, z^{j_{1} \ldots j_{n}}=\min _{\forall j}\left(\mu_{A_{j}^{j_{1} \ldots j_{n}}}\left(x_{j}\right)\right)$,

where $\mu_{A_{1}^{j_{1} \ldots j_{n}}}$ is declared by triangular MF, and then we obtain:

$z^{j_{1} \ldots j_{n}}=\min _{\forall j}\left(\max \left(\min _{\forall k}\left(\frac{x_{k 0}^{p}-a_{k}^{j_{1} \ldots j_{n}}(q)}{b_{k}^{j_{1} \ldots j_{n}}(q)-a_{k}^{j_{1} \ldots j_{n}}(q)}, \frac{c_{k}^{j_{1} \ldots j_{n}}(q)-x_{k 0}^{p}}{c_{k}^{j_{1} \ldots j_{n}}(q)-b_{k}^{j_{1} \ldots j_{n}}(q)}\right), \quad 0\right)\right)$

Third output: Depending on (30), the $j_{1} \ldots j_{n}{ }^{\text {th }}$ node calculates all rules as:

$o_{3}^{j_{1} \ldots j_{n}}=\bar{z}^{j_{1} \ldots j_{n}}(x)=\frac{z^{j_{1} \ldots j_{n}}}{\sum_{j_{1}=1}^{N_{1}} \ldots \sum_{j_{n}=1}^{N_{n}} z^{j_{1} \ldots j_{n}}}$

Fourth output: Every node $j_{1} \ldots j_{n}$ is an adaptive node with a node MF of output.

$o_{4}^{j_{1} \ldots j_{n}}=\bar{z}^{j_{1} \ldots j_{n}} \bar{y}^{j_{1} \ldots j_{n}}$

where $\bar{y}^{j_{1} \ldots j_{n}}=f_{j_{1} \ldots j_{n}}\left(x_{1}, x_{2}, \ldots, x_{n}\right)$, and from (9), we get:

$\bar{y}^{j_{1} \ldots j_{n}}=\alpha_{1}^{j_{1} \ldots j_{n}} x_{1}+\alpha_{2}^{j_{1} \ldots j_{n}} x_{2}+\cdots+\alpha_{n}^{j_{1} \ldots j_{n}} x_{n}+\alpha_{n+1}^{j_{1} \ldots j_{n}}$,

where $\alpha_{j}^{j_{1} \ldots j_{n}},(j=1, \ldots, n+1$.), is the parameter set of the node.

Fifth output: The single node is a fixed node labeled $\sum$, which computes the final output as the summation of all result $o_{4}^{j_{1} \ldots j_{n}}$

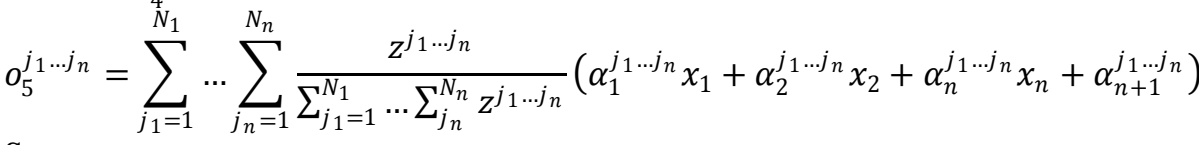

Suppose,

$K=\sum_{\substack{j_{1}=1 \\ N_{1}}}^{N_{1}} \ldots \sum_{\substack{j_{n}=1 \\ N_{n}}}^{N_{n}} z^{j_{1} \ldots j_{n}}$

$L=\sum_{j_{1}=1}^{N_{1}} \ldots \sum_{j_{n}=1}^{N_{n}} z^{j_{1} \ldots j_{n}}\left(\alpha_{1}^{j_{1} \ldots j_{n}} x_{1}+\alpha_{2}^{j_{1} \ldots j_{n}} x_{2}+\alpha_{n}^{j_{1} \ldots j_{n}} x_{n}+\alpha_{n+1}^{j_{1} \ldots j_{n}}\right)$

Consequently, the final output is obtained as:

$f(x)=\frac{L}{K}$.

Here, noted that $\bar{y}^{j_{1} \ldots j_{n}}$ are free parameters to be modeled. When select the initial parameters $\theta(1)$, and there are linguistic rules from experts, then choose $\bar{y}^{j_{1} \ldots j_{n}}(1)$ to be the centers of the then part fuzzy sets in these linguistic rules; otherwise, choose $\theta(1)$ arbitrarily in the output space $Y \subset R$. In this way, we can say that the initial fuzzy system is constructed from experts.

\section{Step3: Update the parameters}

Use the training algorithm to compute the updated parameters $\bar{y}^{j_{1} \ldots j_{n}}(q+1), a_{k}^{j_{1} \ldots j_{n}}(q+1), b_{k}^{j_{1} \ldots j_{n}}(q+1)$, $c_{k}^{j_{1} \ldots j_{n}}(q+1)$, where $y=y_{0}^{p}$, and $z^{j_{1} \ldots j_{n}}, K, L$ and $f$ equal to those that computed in step2, i.e., compute the new parameters $\theta$ using the least squares model as: 
$\theta(p+1)=\theta(p)+t(p+1)\left[y_{0}^{p}-\left(z\left(x_{k 0}^{p}\right)\right)^{T} \theta(p)\right]$

in which,

$t(p+1)=\frac{P(p+1) z\left(x_{k 0}^{p}\right)}{\left[P(p+1) z\left(x_{k 0}^{p}\right)\left(z\left(x_{k 0}^{p}\right)\right)^{T}+1\right]}$,

$P(p+1)=P(p)-\frac{P(p) z\left(x_{k 0}^{p}\right)}{\left[P(p) z\left(x_{k 0}^{p}\right)\left(z\left(x_{k 0}^{p}\right)\right)^{T}+1\right]} P(p)\left(z\left(x_{k 0}^{p}\right)\right)^{T}$

when $p=1$, note that $\theta(1)$ is chosen using step 2 , and $P(1)$ is a large constant. The modeled fuzzy system in (26) with the parameters $\bar{y}^{j_{1} \ldots j_{n}}$ is equal to the corresponding elements in $\theta(p)$.

Step4: Repeat by going to step 2 with $q=q+1$, until the error $\left|f-y_{0}^{p}\right|<\varepsilon$, or until the q equals a prespecified number.

Step5: Repeat by going to step 2 with $p=p+1, p=1,2, \ldots$; that is, update the parameters using the next input-output pair $\left(x_{k 0}^{p+1} ; y_{0}^{p+1}\right)$.

Keep in mind that the parameters $\overline{\mathrm{y}}^{\mathrm{j}_{1} \ldots \mathrm{j}_{\mathrm{n}}}$ are the centers of the fuzzy sets in the consequent parts of the rules, and the parameters $a_{j}^{j_{1} \ldots j_{n}}$ and $c_{j}^{j_{1} \ldots j_{n}}$ are the left and right base points, $b_{j}^{j_{1} \ldots j_{n}}$, the centers of the triangular fuzzy sets in the antecedent parts of the rules [Rojas (1996)]. We can improve the fuzzy if-then rules that modeled the fuzzy system, and improved fuzzy if-then rules may help to explain the model fuzzy system in a user-friendly manner [Aik and Jayakumar (2008)].

\section{Application}

Liver is the largest internal member in the human body, and it is known that the member is responsible for more than one hundred functions of human body. The complexity of this member makes it easily affected by disease of disorder. Therefore, diagnosing liver disorder disease (LDD) is a high interest to researchers and doctors [1], and fuzzy system has been a good intelligent model to diagnose such disease [Sug (2012) and Gulia et al. (2014)]. The fuzzy system has very good property that the model is easy to understand. This property of fuzzy system is important in case that human should understand the knowledge structures fully. This is one of the main reasons why fuzzy system is accepted in medical domain. There are six continuous attributes as dependent attributes, (Table 1 for detail of the attributes). The first five variables are all blood tests that are thought to be sensitive to liver disorders that might result from excessive alcohol consumption. Each line in the LDD_data constitutes the record of a single male individual.

Table 1: The meaning of variables

\begin{tabular}{|c|l|l|l|}
\hline Variable & $\begin{array}{c}\text { Variable } \\
\text { name }\end{array}$ & Meaning & Range \\
\hline$x_{1}$ & mcv & mean corpuscular volume & {$[79,103]$} \\
\hline$x_{2}$ & alkphos & alkaline phosphotase & {$[35,109]$} \\
\hline$x_{3}$ & sgpt & alamine aminotransferase & {$[5,155]$} \\
\hline$x_{4}$ & sgot & aspartate aminotransferase & {$[11,68]$} \\
\hline$x_{5}$ & gammagt & gamma-glutamyl transpeptidase & {$[5,297]$} \\
\hline$x_{6}$ & drinks & number of half-pint equivalents of alcoholic beverages drunk per day & {$[0,20]$} \\
\hline
\end{tabular}

\subsection{Structure of the Model}

Consider the multi-inputs $x_{i},(i=1,2, \ldots, 6)$, with output y (disorder types of liver that contains simple liver disorder or acute liver disorder). A fuzzy inference system (FIS) can be defined as:

FIS: $X \rightarrow Y$, where $X \subset R^{n}$ and $Y \subset R$.

The fuzzy system is composed of a fuzzifier, fuzzy rule-base, fuzzy inference, and defuzzifier. In order to apply a steps of FIS systematically, the inputs $x_{1}, x_{2}, \ldots, x_{6}$ with output $y$ must be described as the following: 


\section{Inputs}

Inputs-data have treated and measured, and it becomes restricted between zero and one. The first five variables have five different degrees of linguistic variables: Low (L), Low Medium (LM), Medium (M), High Medium (HM) and High $(\mathrm{H})$, while sixth input is represented by five linguistic variables, Less (Le), Less Average (LA), Average (A), More Average (MA) and More (Mo).

\section{Output}

Output-data are represented the disorder types of liver that contains two types: simple liver disorder (SLD) or acute liver disorder (ALD), see Figure 2.

\subsection{Discussion and Results of the Fuzzy System with Mamdani and ST Models} In this part, we build the proposed fuzzy system step by step on our application:

\section{Step 1:}

Define $N_{j},(j=1,2, \ldots, 6)$, where $N_{j}=1,2,3$. Let $A_{i}^{1}, \ldots, A_{i}^{6}$ is a fuzzy sets for $x_{i}$, with triangular MFs, $\mu_{A_{1}^{1}}\left(x_{1} ; a_{1}^{1}, b_{1}^{1}, c_{1}^{1}\right), \ldots, \mu_{A_{6}^{5}}\left(x_{6} ; a_{6}^{5}, b_{6}^{5}, c_{6}^{5}\right)$, and $A_{j}^{1}<A_{j}^{2}<\cdots<A_{j}^{N_{j}}$ with $a_{j}^{1}=b_{j}^{1}=0$ and $b_{j}^{N_{j}}=c_{j}^{N_{j}}=1$.

Therefore, we can define:

$e_{1}^{1}=0, e_{1}^{5}=1$, and $e_{1}^{2}=b_{1}^{2}, e_{1}^{3}=b_{1}^{3}, e_{1}^{4}=b_{1}^{4}$
$e_{2}^{1}=0, e_{2}^{5}=1$, and $e_{2}^{2}=b_{2}^{2}, e_{2}^{3}=b_{2}^{3}, e_{2}^{4}=b_{2}^{4}$,
$e_{3}^{1}=0, e_{3}^{5}=1$, and $e_{3}^{2}=b_{3}^{2}, e_{3}^{3}=b_{3}^{3}, e_{3}^{4}=b_{3}^{4}$,
$\quad \quad e_{4}^{1}=0, e_{4}^{5}=1$, and $e_{4}^{2}=b_{4}^{2}, e_{4}^{3}=b_{4}^{3}, e_{4}^{4}=b_{4}^{4}$,
$\quad e_{5}^{1}=0, e_{5}^{5}=1$, and $e_{5}^{2}=b_{5}^{2}, e_{5}^{3}=b_{5}^{3}, e_{5}^{4}=b_{5}^{4}$,
$e_{6}^{1}=0, e_{6}^{3}=1$, and $e_{6}^{2}=b_{6}^{2}$.

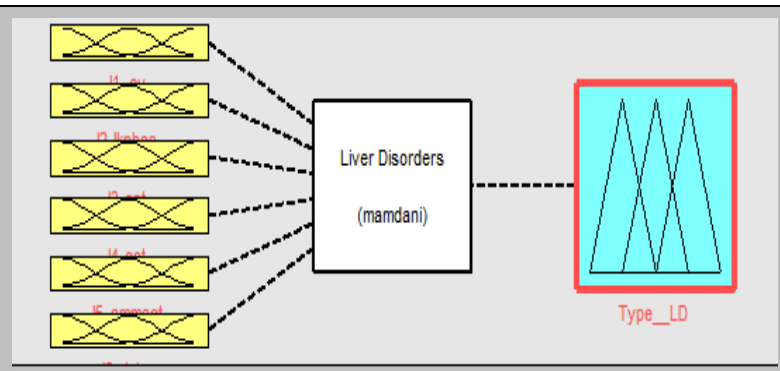

Figure 2a: The FIS of LD using Mamdani model

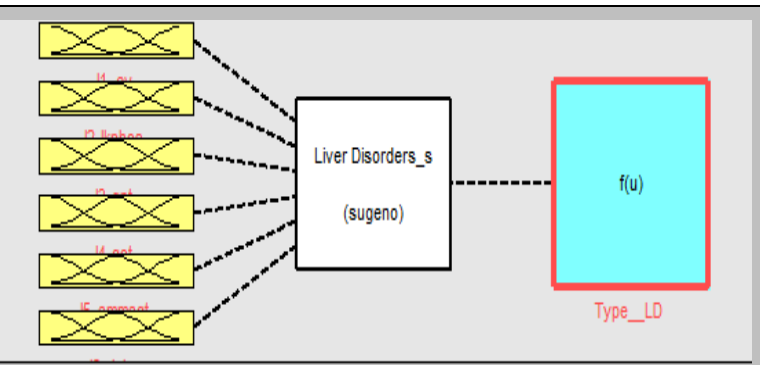

Figure 2b: The FIS of LD using ST model

Here the Singleton fuzzifier is defined as: $S F: x^{*} \rightarrow A^{\prime}$, where $x^{*}=\left\{x_{1}, \ldots, x_{6}\right\}$, and the softwaer 'MATLAB' is used to create the programs of our application. Six vectors of inputs with one vector of output are loaded for 100 observations. For example, the first observation is represented as $[\mathrm{mcv}=0.8544$, alkphos $=0.5088$, $\mathrm{sgpt}=0.12903$, sgot $=0.25$, gammagt $=0.0303$, and drinks $=0.025$ ]. Consequently, we have built the first rule such as [If ( $\mathrm{mcv}$ is $\mathrm{M}$ ) and (alkphos is M) and (sgpt is L) and (sgot is LM) and (gammagt is L) and (drinks is L) Then y is SLD].

\section{Step2:}

Note that, the fuzzy rule-base consists of $\left(I=5^{6}\right)$ fuzzy if-then rules and the centers of $\left(f^{j_{1} \ldots j_{6}, i}\left(x_{1}, \ldots, x_{6}\right)\right)$ are evaluated at the 729 points, and therefore, we obtain:

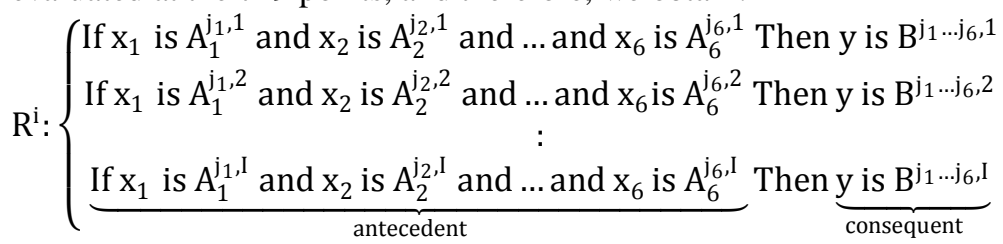

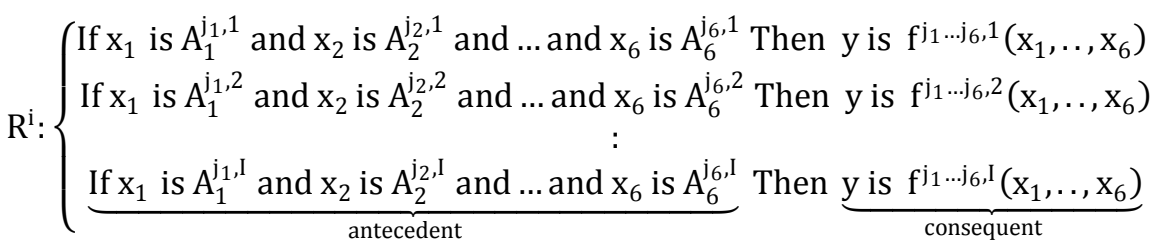


The model (42a) is a system of Mamdani fuzzy rules, while the model (42b) is a system of Sugeno fuzzy rules. The sets A and B are a fuzzy sets, $x_{h}(h=1, \ldots, 6)$ are an input variables, $\mathrm{y}$ is the output variable, and $i,(i=1, \ldots, I)$, is the number of rules. The fuzzy set A consists of, $\left(A_{1}^{j_{1}}, \ldots, A_{6}^{j_{6}}\right)$, fuzzy subsets. It is called linguistic terms that represented by triangular MFs such as (1) with $\mathrm{b}=\mathrm{c}$. The fuzzy operator "and" (t-norm) is used to connecting between linguistic terms in each rule of the model. The function $f^{j_{1} \ldots j_{6}, i}\left(x_{1}, \ldots, x_{6}\right)$ is a linear function depends on inputs $x_{k}$ that defined using (9). The first five linguistic terms are represented by $A_{h}^{j_{i}}\left(j_{i}=\right.$ $1,2, \ldots, 5)$ that depends on linguistic variable $x_{h}(h=1, \ldots, 5)$, see Figure 3, for e.g. the linguistic term for first input variable defined as the following:

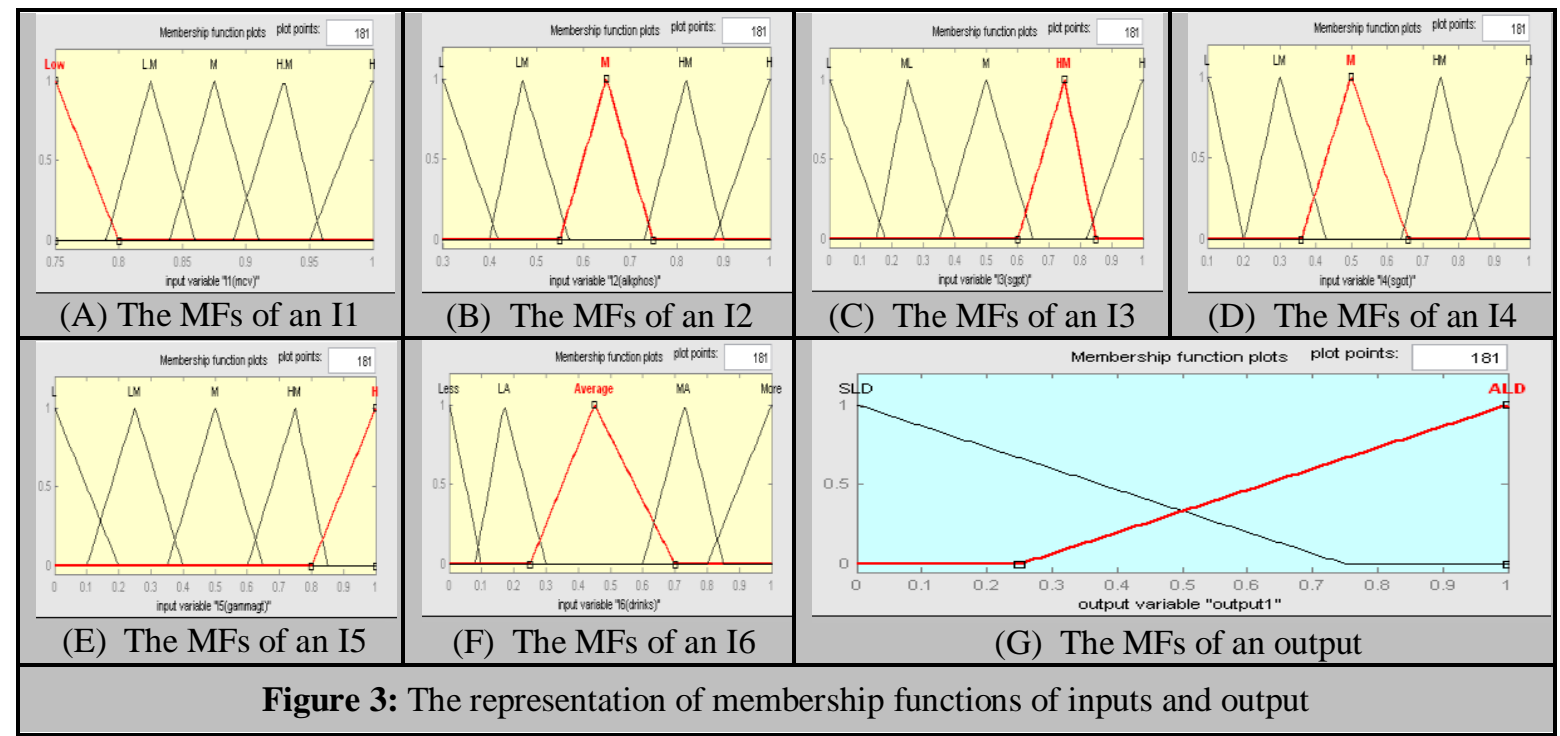

$A_{1}^{1} \equiv$ "Low" $\equiv\left(\mu_{A_{1}^{1}}\left(x_{1} ; 0,0,0.18\right)\right), \mathrm{A}_{1}^{2} \equiv$ "Low Mediam" $\equiv\left(\mu_{A_{1}^{2}}\left(x_{1} ; 0.15,0.25,0.4\right)\right), A_{1}^{3} \equiv$ "Mediam", $\equiv$ $\left(\mu_{A_{1}^{3}}\left(x_{1} ; 0.35,0.25,0.65\right)\right), A_{1}^{4} \equiv$ "High Medium" $\equiv\left(\mu_{A_{1}^{4}}\left(x_{1} ; 0.6,0.73,0.85\right)\right)$ and $A_{1}^{5} \equiv$ "High" $\equiv\left(\mu_{A_{1}^{5}}\left(x_{1} ; 0.82,1,1\right)\right)$. While, we have represented the sixth linguistic term $A_{6}^{j_{i}}\left(j_{i}=1, \ldots, 5\right)$ that depends on linguistic variable $x_{6}$ as the following:

$A_{6}^{1} \equiv$ "Less" $\equiv\left(\mu_{A_{6}^{1}}\left(x_{6} ; 0,0,0.1\right)\right), A_{6}^{2} \equiv$ "Less Average" $\equiv\left(\mu_{A_{6}^{2}}\left(x_{6} ; 0.08,0.2,0.3\right)\right), A_{6}^{3} \equiv$ “Average"

$\equiv\left(\mu_{A_{6}^{3}}\left(x_{6} ; 0.25,0.5,0.7\right)\right), A_{6}^{4} \equiv$ "More Average" $\equiv\left(\mu_{A_{6}^{4}}\left(x_{6} ; 0.6,0.7,0.83\right)\right)$ and $A_{6}^{5} \equiv$ "More" $\equiv\left(\mu_{A_{6}^{5}}\left(x_{6} ; 0.8,1,1\right)\right)$.

Moreover, the output is described by triangular MFs, $\left(\mu_{B^{j_{1} \ldots j_{6}}}(y)\right)$, and its linguistic term that represented as the following:

$B^{1} \equiv " \operatorname{SLD} " \equiv\left(\mu_{B}(y ; 0,0,0.75)\right)$ and $B^{2} \equiv “ \operatorname{ALD} " \equiv\left(\mu_{B} 2(y ; 0.25,1,1)\right)$.

The fuzzy inference process defines as the following:

$F I: A^{\prime} \rightarrow \overline{B^{j 1 \ldots j} j_{6}, i}$,

where $A^{\prime}$ is an input fuzzy set in the input space $X$, and $\overline{B^{j_{1} \ldots j_{6}, i}}$ the fuzzy sets in the output space $Y$. Each one of the rules specifies a fuzzy set $\overline{B^{j_{1} j_{2} j_{3}, i}} \subseteq Y$ that is given by the compositional rule of inference:

$\overline{B^{j_{1} \ldots j_{6}, i}}=A^{\prime} \circ\left(A_{j}^{j_{1} \ldots j_{6}, i} \rightarrow B^{j_{1} \ldots j_{6}, i}\right)$, where $A_{j}^{j_{1} \ldots j_{6}, i}=A_{1}^{j_{1} \ldots j_{6}, i} \times A_{2}^{j_{1} \ldots j_{6}, i} \times \ldots \times A_{6}^{j_{1} \ldots j_{6}, i}$

Therefore, from $\mu_{A_{1}^{i} \times \ldots \times A_{n}^{i}}\left(x_{1}, \ldots, x_{n}\right)=\mu_{A_{1}^{i}}\left(x_{1}\right) * \ldots * \mu_{A_{n}^{i}}\left(x_{n}\right)$, we obtain

$\mu_{A_{j}^{j} j_{1} \ldots j_{6}, i}\left(x_{j}\right)=\mu_{A_{1}^{j 1} \ldots j_{6}, i} \times A_{2}^{j_{1} \ldots j_{6}, i} \times \ldots \times A_{6}^{j_{1} \ldots j_{6}, i}\left(x_{1}, \ldots, x_{6}\right),(j=1,2, \ldots, 6)$

From $\left(\mu_{B^{\prime}}(y)=\sup _{x \in X}\left\{\mu_{A^{\prime}}(x) \stackrel{t}{+} \mu_{Q_{I}}(x, y)\right\}\right)$, the fuzzy sets $\overline{B^{j 1_{1} \ldots j_{6}, i}}$ are described by MF:

$\mu_{B^{j 1 \ldots j}, i}(y)=\sup _{x \in X}\left\{\mu_{A^{\prime}}(x)_{\dot{+}}^{t} \mu_{\left(A_{j}^{j_{1} \ldots j_{6}, i} \rightarrow B^{j 1 \ldots j}, i\right.}(x, y)\right\}$

Consequently, can be re-express (46) as the following

$\mu_{B^{j 1 \ldots j}, i}(y)=\mu_{A_{j}^{j_{1} \ldots j_{6}, i} \rightarrow B^{j_{1} \ldots j_{6}, i}}\left(x_{j}, y\right)=\operatorname{Im}\left(\mu_{A_{j}^{j_{1} \ldots j_{6}, i}}\left(x_{j}\right), \mu_{B^{j_{1} \ldots j_{6}, i}}(y)\right)$,

where $\operatorname{Im}($.$) is an "implementation”. Since, we used Mamdani's minimum implication (10a), therefore, we$ obtained: 
$\operatorname{Im}\left(\mu_{A_{j}^{j_{1} \ldots j_{6}, i}}\left(x_{j}\right), \mu_{B^{j_{1} \ldots j_{6}, i}}(y)\right)=\min \left\{\mu_{A_{j}^{j_{1} \ldots j_{6}, i}}\left(x_{j}\right), \mu_{B^{j_{1} \ldots j_{6}, i}}(y)\right\}$

or (47) may be written as:

$\mu_{\overline{B^{j 1} \ldots \sigma_{6}, i}}(y)=\min \left\{\mu_{A_{j}^{j_{1} \ldots j_{6}, i}}\left(x_{j}\right), \mu_{B^{j_{1} \ldots j_{6}, i}}(y)\right\}$

The aggregation operator has been applied in order to get the fuzzy set $B$ ' that uses the functions ' $\max$ ' (s-norm) or ' $\min$ ' (t-norm) depending on the type of fuzzy implication. The aggregation operator is denoted by:

$B^{\prime}=\bigcup_{i=1}^{I} \overline{B^{j} \ldots j_{6}, i}$,

Therefore, the membership function of $\mathrm{B}^{\prime}$ is computed using the ' $m a x$ ' function as the following:

$\mu_{B^{\prime}}(y)=\max _{\forall i}\left\{\mu_{B^{j / 1 / 2 j, i}}(y)\right\}$

\section{Stepe3:}

The defuzzifier performs a mapping as the following:

def $=B^{\prime} \rightarrow f(x)$,

where $B^{\prime}$ is a fuzzy set, $f(x)$ is a crisp point $f(x) \in(Y \subset R)$. The center of the area is a final system output that is defined by the following formula:

$$
f(x)=\frac{\sum_{j_{1}=1}^{5} \ldots \sum_{j_{6=1}}^{5} \bar{y}^{j_{1} \ldots j_{6}}\left(\min \left(\max \left(\min _{j=1: 6}\left(\frac{x-a_{j}^{j_{1} \ldots j_{6}}}{b_{j}^{j_{1} \ldots j_{6}}-a_{j}^{j_{1} \ldots j_{6}}}, \frac{c_{j}^{j_{1} \ldots j_{6}}-x}{c_{j}^{j_{1} \ldots j_{6}}-b_{j}^{j_{1} \ldots j_{6}}}\right), 0\right)\right)\right)}{\sum_{j_{1}=1}^{5} \ldots \sum_{j_{6=1}}^{5}\left(\min \left(\max \left(\min _{j=1: 6}\left(\frac{x-a_{j}^{j_{1} \ldots j_{6}}}{b_{j}^{j_{1} \ldots j_{6}}-a_{j}^{j_{1} \ldots j_{6}}}, \frac{c_{j}^{j_{1} \ldots j_{6}}-x}{c_{j}^{j_{1} \ldots j_{6}}-b_{j}^{j_{1} \ldots j_{6}}}\right), 0\right)\right)\right)}
$$

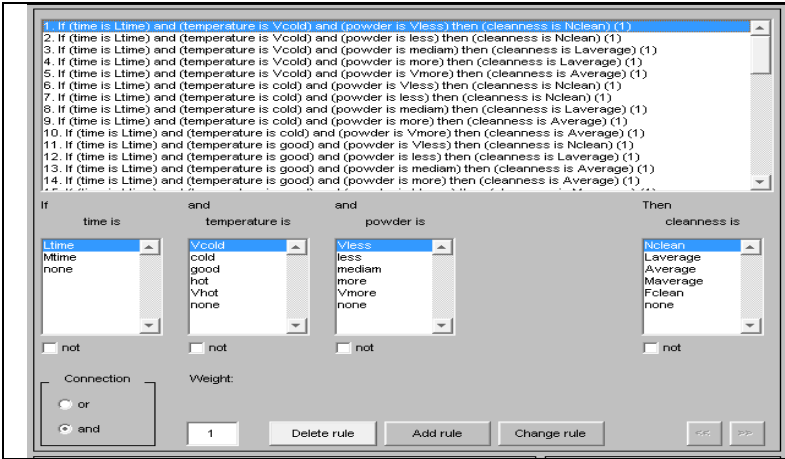

Figure 4a: Representation of the rules editor

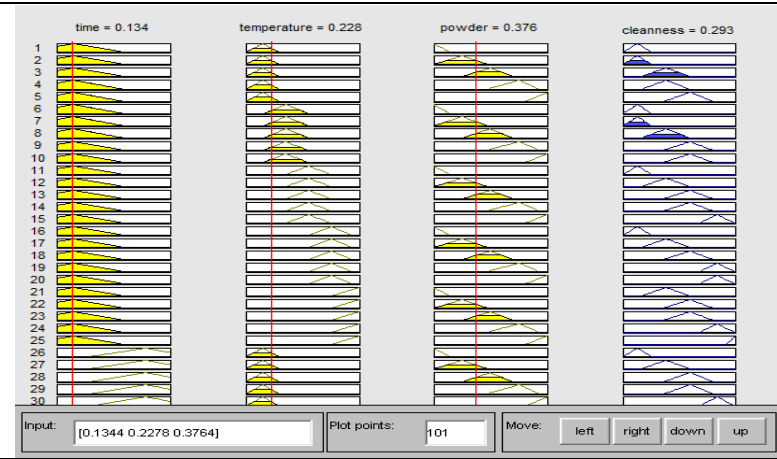

Figure 4b: The rule view of the FIS

The model of fuzzy system $\mathrm{f}(\mathrm{x})$ has been built from the $I$ rules of (42a) using Mamdani's minimum implication (10a) with the MIS (10b), the SF (11), and the CAD (12) (see Figure 4). We have created two programs; the first program depends on the Mamdani model, while the second program depends on the ST model. The programs of FIS have applied, that is given by (52), and obtained a good result of target. Therefore, we have applied the measure for accuracy of all data. The deference between the actual and target outputs is given by the formula as the following:

Error $=|R v-F I S v|$,

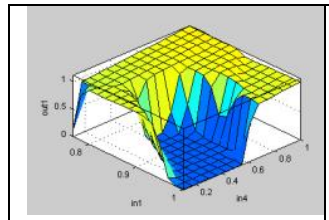

(a) between I1 \& I4

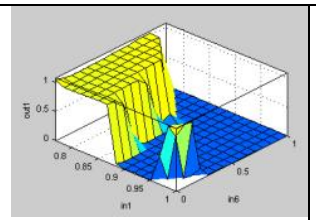

(b) between I1 \& I6

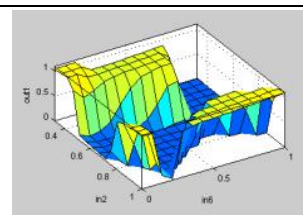

(c) between I6 \& I2

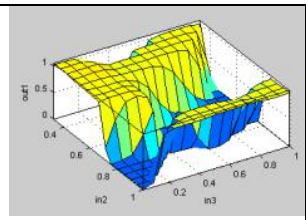

(d) between I2 \& I3

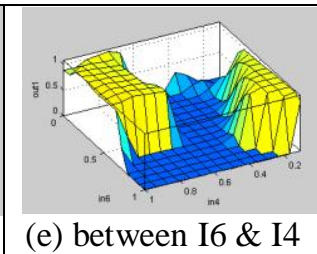

(e) between I6 \& I4

Figure 5: The output surface for the different inputs 
where $R v$ is actual output values and FISv is the output target values. The value of accuracy is a very small, where the average error of Simple $\mathrm{LD}=0.2047$ and the average error of Acute $\mathrm{LD}=0.1158$, when FIS is used the Mamdani fuzzy rule model. While, when the FIS is used the ST fuzzy rule model, then the average error of Simple $\mathrm{LD}=0.1463$ and the average error of Acute $\mathrm{LD}=0.0061$. Table 2 represents all the results. The 'surf view' tool is the surface viewer that helps view the input-output surface of the FIS. This conception is very helpful to understand how the system is going to behave for the entire range of values in the inputs space. Figure 5 shows the output surface for the different inputs.

\subsection{Discussion and Results of the Adaptive Fuzzy System Using Neural Network}

In this Section, specify the structure of the fuzzy system to be modeled. Here, we choose the fuzzy system with a MIS, a SF, the CAD, and a Triangular MF that given by the model (52). Model (52) has not been modeled, because the free parameters $\bar{y}^{j_{1} \ldots j_{6}}, a_{j}^{j_{1} \ldots j_{6}}, b_{j}^{j_{1} \ldots j_{6}}$, and $c_{j}^{j_{1} \ldots j_{6}}$ are not specified. These parameters should be determined in order to represent the $f(x)$. The Model of the adaptive fuzzy system using neural network may be express as the following:

Step1: Determine the initial parameters $\bar{y}^{j_{1} \ldots j_{6}}(0), a_{j}^{j_{1} \ldots j_{6}}(0), b_{j}^{j_{1} \ldots j_{6}}(0)$, and $c_{j}^{j_{1} \ldots j_{6}}(0)$ according to the linguistic rules from experts, such as when $j=j_{h}=1,(h=1, \ldots, 6)$, then $a_{1}^{1}(0)=0, b_{1}^{1}(0)=0, c_{1}^{1}(0)=$ 0.18 , similarly for $\forall j_{h}$, and $\mathrm{j}$ and for each input.

Step2: From a given inputs-output pair $\left(x_{1,0}^{p}, \ldots, x_{6,0}^{p} ; y_{0}^{p}\right), \forall p$ (observe), compute the outputs of layers as the following:

(i) The node of the first output $o_{1}^{j_{\mathrm{i}}}$ is represented by:

$o_{1}^{j_{1}}=\mu_{A_{1}^{1}}\left(x_{1}\right),\left(j_{1}=1, \ldots, 5\right) ; o_{1}^{j_{2}}=\mu_{A_{1}^{1}}\left(x_{1}\right),\left(j_{2}=5, \ldots, 10\right) ; o_{1}^{j_{3}}=\mu_{A_{1}^{1}}\left(x_{1}\right),\left(j_{3}=10, \ldots, 15\right) ; o_{1}^{j_{4}}=$

$\mu_{A_{1}^{1}}\left(x_{1}\right),\left(j_{4}=15, \ldots, 20\right) ; o_{1}^{j_{5}}=\mu_{A_{1}^{1}}\left(x_{1}\right),\left(j_{5}=20, \ldots, 25\right)$ and $o_{1}^{j_{6}}=\mu_{A_{1}^{1}}\left(x_{1}\right),\left(j_{6}=25, \ldots, 30\right)$,

where $o_{1}^{j_{i}}$ is the degree of MFs of a fuzzy set.

(ii) To compute the second output, we should use the minimum function of all MFs as:

$o_{2}^{j_{1} \ldots j_{6}}=\min _{\forall j_{1} \ldots j_{6}}\left(\mu_{A_{1}^{j_{1} \ldots j_{6}}}\left(x_{1}\right), \mu_{A_{2}^{j_{1} \ldots j_{6}}}\left(x_{2}\right), \ldots, \mu_{A_{6}^{j_{1} \ldots j_{6}}}\left(x_{6}\right)\right)$.

Since $\mu_{A_{1}^{j} j_{1} \ldots j_{6}}$ is a Triangular MF, therefore, we obtain:

$o_{2}^{j_{1} \ldots j_{6}}=\min _{\forall j_{1} \ldots j_{6}}\left(\max \left(\min _{\forall k}\left(\frac{x_{k 0}^{p}-a_{k}^{j_{1} \ldots j_{6}}(q)}{b_{k}^{j_{1} \ldots j_{6}}(q)-a_{k}^{j_{1} \ldots j_{6}}(q)}, \frac{c_{k}^{j_{1} \ldots j_{6}}(q)-x_{k 0}^{p}}{c_{k}^{j_{1} \ldots j_{6}}(q)-b_{k}^{j_{1} \ldots j_{6}}(q)}\right), 0\right)\right)$.

(iii) For all rules, we have calculated the $j_{1} \ldots j_{6}{ }^{\text {th }}$ node as:

$$
o_{3}^{j_{1} \ldots j_{6}}=\left(\frac{\min _{\forall j}\left(\max \left(\min _{\forall k}\left(\frac{x_{k 0}^{p}-a_{k}^{j_{1} \ldots j_{6}}(q)}{b_{k}^{j_{1} \ldots j_{6}}(q)-a_{k}^{j_{1} \ldots j_{6}}(q)}, \frac{c_{k}^{j_{1} \ldots j_{6}}(q)-x_{k 0}^{p}}{c_{k}^{j_{1} \ldots j_{6}}(q)-b_{k}^{j_{1} \ldots j_{6}}(q)}\right), 0\right)\right)}{\sum_{j_{1}=1}^{5} \ldots \sum_{j_{6}}^{5} \min _{\forall j}\left(\max \left(\min _{\forall k}\left(\frac{x_{k 0}^{p}-a_{k}^{j_{1} \ldots j_{6}}(q)}{b_{k}^{j_{1} \ldots j_{6}}(q)-a_{k}^{j_{1} \ldots j_{6}}(q)}, \frac{c_{k}^{j_{1} \ldots j_{6}}(q)-x_{k 0}^{p}}{c_{k}^{j_{1} \ldots j_{6}}(q)-b_{k}^{j_{1} \ldots j_{6}}(q)}\right), 0\right)\right)}\right) .
$$

(iv) The fourth output depends on a node MF of output $\bar{z}^{j_{1} \ldots j_{6}}$ with an adaptive node $\bar{y}^{j_{1} \ldots j_{6}}$, therefore, we obtain

$o_{4}^{j_{1} \ldots j_{6}}=o_{3}^{j_{1} \ldots j_{6}} f_{j_{1} \ldots j_{6}}\left(x_{1}, x_{2}, \ldots, x_{6}\right)$

Since $f_{j_{1} \ldots j_{6}}\left(x_{1}, x_{2}, \ldots, x_{6}\right)=\alpha_{1}^{j_{1} \ldots j_{6}} x_{1}+\alpha_{2}^{j_{1} \ldots j_{6}} x_{2}+\cdots+\alpha_{6}^{j_{1} \ldots j_{6}} x_{6}+\alpha_{7}^{j_{1} \ldots j_{6}}$, then we should determine the initial parameters of $\left(\alpha_{j}^{j_{1} \ldots j_{6}}, \forall j=1, \ldots, 7.\right)$.

(v) In order to compute the final output, we must take the summation of all results $o_{4}^{j_{1} \ldots j_{6}}$ as the following:

$o_{5}^{j_{1} \ldots j_{6}}=\sum_{j_{1}=1}^{5} \ldots \sum_{j_{6}=1}^{5} \frac{o_{2}^{j_{1} \ldots j_{6}}}{\sum_{j_{1}=1}^{5} \ldots \sum_{j_{6}}^{5} o_{2}^{j_{1} \ldots j_{6}}} *\left(\alpha_{1}^{j_{1} \ldots j_{6}} x_{1}+\alpha_{2}^{j_{1} \ldots j_{6}} x_{2}+\cdots+\alpha_{6}^{j_{1} \ldots j_{6}} x_{6}+\alpha_{7}^{j_{1} \ldots j_{6}}\right)$ 
Step3: In step 2, it is specified the initial parameters of $\bar{y}^{j_{1} \ldots j_{6}}(q), a_{k}^{j_{1} \ldots j_{6}}(q), b_{k}^{j_{1} \ldots j_{6}}(q), c_{k}^{j_{1} \ldots j_{6}}(q)$ and $q=0$. Consequently, we update these parameters at $q+1$, using the least squares model and repeat all the procedure in step 2 for compute $K, L$ and $f$. From (37) to (39), we compute the new parameters as follows:

$$
\begin{aligned}
& \theta(2)=\theta(1)+t(2)\left[0.462-\left(o_{2}^{j_{1} \ldots j_{6}}\right)^{T} \theta(1)\right] \\
& t(2)=\frac{P(2) o_{2}^{j_{1} \ldots j_{6}}}{\left[P(2) o_{2}^{j_{1} \ldots j_{6}}\left(o_{2}^{j_{1} \ldots j_{6}}\right)^{T}+1\right]} \\
& P(2)=1-\frac{P(1) o_{2}^{j_{1} \ldots j_{6}}}{\left[P(1) o_{2}^{j_{1} \ldots j_{6}}\left(o_{2}^{j_{1} \ldots j_{6}}\right)^{T}+1\right]} 1 *\left(o_{2}^{j_{1} \ldots j_{6}}\right)^{T}
\end{aligned}
$$

Similarly, we can update all parameters of $\theta$ for all training data. Repeat all procedures in step 2 with $q=q+$ 1 ttil the last specific number of $q$ is reached.

From the program of the AFS using a Neural Network that is given by model (52), we have obtained better results with the AFS using a Neural Network than the result of the FIS. We have applied the measure of accuracy for all data, and obtained the average error of Simple $\mathrm{LD}=0.000943$, while the average error of Acute $\mathrm{LD}=0.0055$, (see Table 2). Additionally, obtained the best average testing error of training data (0.00000679) with epoch 100, and the average testing error of checking data (0.03802), see Figure 6. As well as, Table 3 presents the representation of results of the FIS with Mamdani model, the FIS with ST models, and the ANFIS with their errors through Appendix.

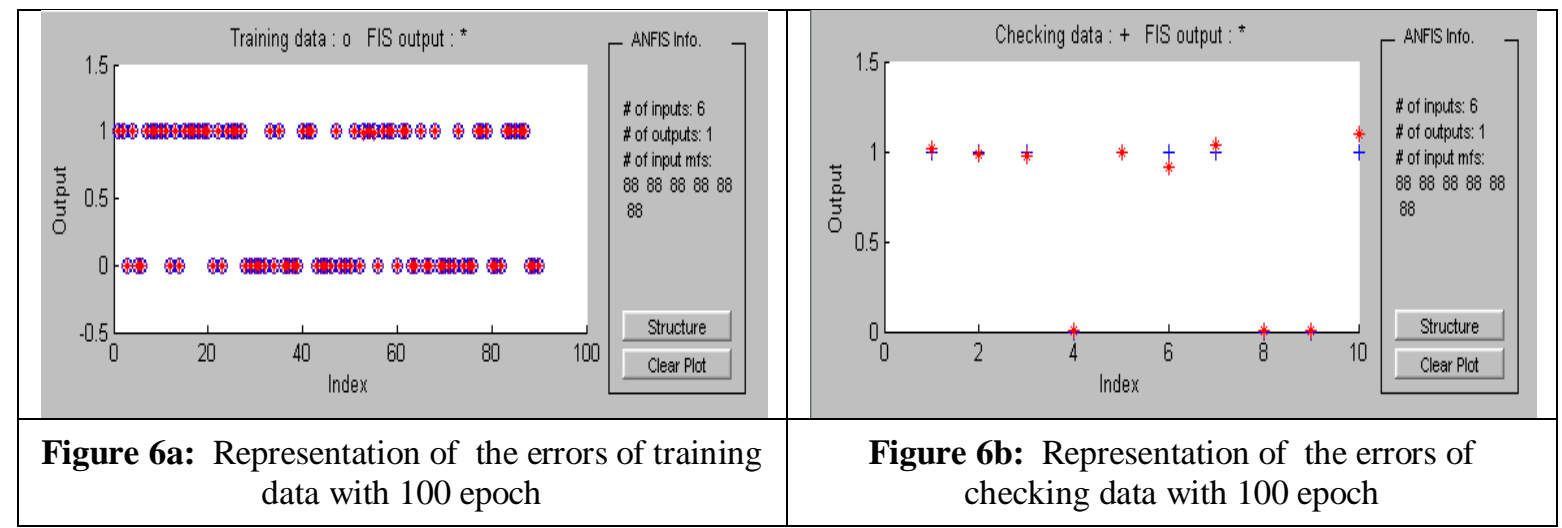

Table 2: Representation errors of Simple, and Acute Liver Disorder and average error for different models.

\begin{tabular}{|c|c|c|c|}
\hline Type of model & $\begin{array}{c}\text { Average error } \\
\text { of Simple LD }\end{array}$ & $\begin{array}{c}\text { Average error } \\
\text { of Acute LD }\end{array}$ & $\begin{array}{c}\text { Average error } \\
\text { of training data }\end{array}$ \\
\hline FIS (Mamdani model) & 0.2047 & 0.1158 & 0.1603 \\
\hline FIS (ST model) & 0.1463 & 0.0061 & 0.0762 \\
\hline ANFIS (ST model) & 0.000943 & 0.0055 & 0.0032 \\
\hline
\end{tabular}

\section{Conclusion}

This work focused on how to use the fuzzy models to solving fuzzy mathematics problems. Here constructed two different models, namely fuzzy inference system, and adaptive neuro-fuzzy inference system. Further, suggested an extension FS and ANFS at N-dimension those depended on the MMI with the MIS, the $\mathrm{SF}$, and the CAD. It is provided the theorem for accuracy of proposed models, as well as, adapted the model of an adaptive FS using a neural network. In addition, we have provided a medical application of the fuzzy mathematics models. We have diagnosed the liver disorder disease that is a high interest to researchers of fuzzy modeling and fuzzy system because the liver is the largest internal member in the human body. Therefore, we have applied the fuzzy mathematical models on the real data to the liver disorder disease. We have presented discussion and results for the model of the FS with Mamdani and ST models, and the ANFIS, respectively. Additionally, we have used the software 'MATLAB' in order to perform the results of different models. Consequently, we have gotten good results for accuracy of these models. The comparison between the three models the FS with Mamdani model, the ST model, and the ANFIS had presented. We have obtained the best 
result with the ANFIS. Finally, we have presented the representation of results of the different models with their errors through Appendix. In the future work, we can develop these models of fuzzy system to generate many outputs or extending the number of input variables. As well as, we can change the fuzzy inference system with another types, or with different type of fuzzifier or defuzzifier.

\section{Acknowledgements}

The author wishes to thank the reviewers for their excellent suggestions that have been incorporated into this paper. I would like to acknowledge my profound gratitude to my research supervisors DR. Sanjeev Kumar, a well-known mathematician and researcher and DR. M. S. Saroa for their vigilant supervision as well as for pouring many invaluable pearls from the deep ocean of knowledge. No volume of words is enough to express my gratitude towards my guide.

\section{APENDIX}

In this Section, present the representation of results of the FIS with Mamdani model, the FIS with ST models, and the ANFIS with their errors.

Table 3: Representation of results of FIS and ANFIS with their errors

\begin{tabular}{|c|c|c|c|c|c|c|c|}
\hline N0. & Real & $\begin{array}{c}\text { FIS with } \\
\text { (Mamdani model) }\end{array}$ & $\mathrm{E}\left(\mathrm{FIS}_{\mathrm{M}}\right)$ & $\begin{array}{c}\text { FIS with } \\
\text { ( ST model) }\end{array}$ & $\mathrm{E}\left(\mathrm{FIS}_{\mathrm{ST}}\right)$ & $\begin{array}{l}\text { ANFIS with } \\
\text { (ST model) }\end{array}$ & $\mathrm{E}\left(\mathrm{ANFIS}_{\mathrm{ST}}\right)$ \\
\hline 1. & 1 & 0.915 & 0.085 & 1.27 & -0.27 & $1.00 \mathrm{E}+00$ & $0.00 \mathrm{E}+00$ \\
\hline 2. & 1 & 0.916 & 0.084 & 1.24 & -0.24 & $1.00 \mathrm{E}+00$ & $0.00 \mathrm{E}+00$ \\
\hline 3. & 0 & 0.197 & -0.197 & 0.225 & -0.225 & $0.00 \mathrm{E}+00$ & $0.00 \mathrm{E}+00$ \\
\hline 4. & 0 & 0.222 & -0.222 & 0.202 & -0.202 & $0.00 \mathrm{E}+00$ & $0.00 \mathrm{E}+00$ \\
\hline 5. & 0 & 0.186 & -0.186 & 0.2 & -0.2 & $0.00 \mathrm{E}+00$ & $0.00 \mathrm{E}+00$ \\
\hline 6. & 1 & 0.907 & 0.093 & 1.2 & -0.2 & $1.00 \mathrm{E}+00$ & $0.00 \mathrm{E}+00$ \\
\hline 7. & 0 & 0.2 & -0.2 & 0.192 & -0.192 & $0.00 \mathrm{E}+00$ & $0.00 \mathrm{E}+00$ \\
\hline 8. & 1 & 0.909 & 0.091 & 1.19 & -0.19 & 0.998 & 0.002 \\
\hline 9. & 0 & 0.197 & -0.197 & 0.188 & -0.188 & $3.04 \mathrm{E}-03$ & $-3.04 \mathrm{E}-03$ \\
\hline 10. & 0 & 0.208 & -0.208 & 0.183 & -0.183 & $-5.85 \mathrm{E}-07$ & $5.85 \mathrm{E}-07$ \\
\hline 11. & 0 & 0.238 & -0.238 & 0.177 & -0.177 & $1.81 \mathrm{E}-05$ & $-1.81 \mathrm{E}-05$ \\
\hline 12. & 0 & 0.196 & -0.196 & 0.177 & -0.177 & $0.00 \mathrm{E}+00$ & $0.00 \mathrm{E}+00$ \\
\hline 13. & 0 & 0.208 & -0.208 & 0.172 & -0.172 & 0 & 0 \\
\hline 14. & 1 & 0.912 & 0.088 & 1.17 & -0.17 & 9.99E-01 & $1.00 \mathrm{E}-03$ \\
\hline 15. & 0 & 0.184 & -0.184 & 0.165 & -0.165 & $1.21 \mathrm{E}-06$ & $-1.21 \mathrm{E}-06$ \\
\hline 16. & 1 & 0.187 & 0.813 & 1.16 & -0.16 & $1.00 \mathrm{E}+00$ & $0.00 \mathrm{E}+00$ \\
\hline 17. & 0 & 0.197 & -0.197 & 0.156 & -0.156 & $0.00 \mathrm{E}+00$ & $0.00 \mathrm{E}+00$ \\
\hline 18. & 0 & 0.202 & -0.202 & 0.155 & -0.155 & $-4.40 \mathrm{E}-08$ & $4.40 \mathrm{E}-08$ \\
\hline 19. & 0 & 0.224 & -0.224 & 0.151 & -0.151 & $3.60 \mathrm{E}-07$ & $-3.60 \mathrm{E}-07$ \\
\hline 20. & 0 & 0.205 & -0.205 & 0.151 & -0.151 & $-3.10 \mathrm{E}-08$ & $3.10 \mathrm{E}-08$ \\
\hline 21. & 0 & 0.202 & -0.202 & 0.149 & -0.149 & $4.98 \mathrm{E}-05$ & $-4.98 \mathrm{E}-05$ \\
\hline 22. & 0 & 0.185 & -0.185 & 0.149 & -0.149 & $-1.00 \mathrm{E}-08$ & $1.00 \mathrm{E}-08$ \\
\hline 23. & 0 & 0.205 & -0.205 & 0.148 & -0.148 & $0.00 \mathrm{E}+00$ & $0.00 \mathrm{E}+00$ \\
\hline 24. & 0 & 0.21 & -0.21 & 0.147 & -0.147 & $1.70 \mathrm{E}-08$ & $-1.70 \mathrm{E}-08$ \\
\hline 25. & 0 & 0.197 & -0.197 & 0.144 & -0.144 & $2.06 \mathrm{E}-07$ & $-2.06 \mathrm{E}-07$ \\
\hline 26. & 0 & 0.203 & -0.203 & 0.144 & -0.144 & $2.28 \mathrm{E}-03$ & $-2.28 \mathrm{E}-03$ \\
\hline 27. & 0 & 0.199 & -0.199 & 0.143 & -0.143 & $5.26 \mathrm{E}-04$ & $-5.26 \mathrm{E}-04$ \\
\hline 28. & 0 & 0.197 & -0.197 & 0.141 & -0.141 & $1.20 \mathrm{E}-08$ & $-1.20 \mathrm{E}-08$ \\
\hline 29. & 0 & 0.216 & -0.216 & 0.141 & -0.141 & 0.0384 & -0.0384 \\
\hline 30. & 0 & 0.2 & -0.2 & 0.14 & -0.14 & $-1.10 \mathrm{E}-08$ & $1.10 \mathrm{E}-08$ \\
\hline 31. & 1 & 0.905 & 0.095 & 1.14 & -0.14 & 0.948 & 0.052 \\
\hline 32. & 0 & 0.202 & -0.202 & 0.139 & -0.139 & $-1.10 \mathrm{E}-08$ & $1.10 \mathrm{E}-08$ \\
\hline 33. & 0 & 0.199 & -0.199 & 0.138 & -0.138 & $-7.54 \mathrm{E}-07$ & $7.54 \mathrm{E}-07$ \\
\hline 34. & 0 & 0.196 & -0.196 & 0.138 & -0.138 & 0 & 0 \\
\hline 35. & 0 & 0.185 & -0.185 & 0.136 & -0.136 & $-4.60 \mathrm{E}-08$ & $4.60 \mathrm{E}-08$ \\
\hline 36. & 0 & 0.254 & -0.254 & 0.135 & -0.135 & $-1.07 \mathrm{E}-07$ & $1.07 \mathrm{E}-07$ \\
\hline 37. & 0 & 0.21 & -0.21 & 0.135 & -0.135 & $6.10 \mathrm{E}-08$ & $-6.10 \mathrm{E}-08$ \\
\hline 38. & 0 & 0.2 & -0.2 & 0.134 & -0.134 & $0.00 \mathrm{E}+00$ & $0.00 \mathrm{E}+00$ \\
\hline 39. & 0 & 0.204 & -0.204 & 0.132 & -0.132 & $-3.33 \mathrm{E}-07$ & $3.33 \mathrm{E}-07$ \\
\hline 40. & 0 & 0.192 & -0.192 & 0.131 & -0.131 & $8.91 \mathrm{E}-07$ & $-8.91 \mathrm{E}-07$ \\
\hline 41. & 0 & 0.174 & -0.174 & 0.13 & -0.13 & $9.00 \mathrm{E}-08$ & $-9.00 \mathrm{E}-08$ \\
\hline 42. & 1 & 0.908 & 0.092 & 1.13 & -0.13 & $1.00 \mathrm{E}+00$ & $0.00 \mathrm{E}+00$ \\
\hline 43. & 1 & 0.917 & 0.083 & 1.13 & -0.13 & 0.992 & 0.008 \\
\hline 44. & 1 & 0.908 & 0.092 & 1.13 & -0.13 & $1.00 \mathrm{E}+00$ & $0.00 \mathrm{E}+00$ \\
\hline 45. & 0 & 0.405 & -0.405 & 0.128 & -0.128 & $-2.37 \mathrm{E}-07$ & $2.37 \mathrm{E}-07$ \\
\hline 46. & 0 & 0.205 & -0.205 & 0.126 & -0.126 & $5.00 \mathrm{E}-09$ & $-5.00 \mathrm{E}-09$ \\
\hline
\end{tabular}


Fuzzy mathematical models for the analysis of fuzzy systems with application to liver disorders

\begin{tabular}{|c|c|c|c|c|c|c|c|}
\hline 47. & 0 & 0.204 & -0.204 & 0.126 & -0.126 & $2.48 \mathrm{E}-07$ & $-2.48 \mathrm{E}-07$ \\
\hline 48. & 0 & 0.192 & -0.192 & 0.126 & -0.126 & 0 & 0 \\
\hline 49. & 0 & 0.178 & -0.178 & 0.125 & -0.125 & -0.000193 & 0.000193 \\
\hline 50. & 0 & 0.172 & -0.172 & 0.125 & -0.125 & 0.000215 & -0.000215 \\
\hline 51. & 0 & 0.216 & -0.216 & 0.124 & -0.124 & $2.32 \mathrm{E}-07$ & $-2.32 \mathrm{E}-07$ \\
\hline 52. & 0 & 0.178 & -0.178 & 0.123 & -0.123 & $-2.55 \mathrm{E}-05$ & $2.55 \mathrm{E}-05$ \\
\hline 53. & 0 & 0.17 & -0.17 & 0.122 & -0.122 & $-2.00 \mathrm{E}-08$ & $2.00 \mathrm{E}-08$ \\
\hline 54. & 0 & 0.2 & -0.2 & 0.117 & -0.117 & $4.70 \mathrm{E}-08$ & $-4.70 \mathrm{E}-08$ \\
\hline 55. & 0 & 0.214 & -0.214 & 0.117 & -0.117 & $-4.30 \mathrm{E}-08$ & $4.30 \mathrm{E}-08$ \\
\hline 56. & 0 & 0.192 & -0.192 & 0.116 & -0.116 & $-6.00 \mathrm{E}-07$ & $6.00 \mathrm{E}-07$ \\
\hline 57. & 0 & 0.2 & -0.2 & 0.113 & -0.113 & 4.70E-07 & $-4.70 \mathrm{E}-07$ \\
\hline 58. & 1 & 0.913 & 0.087 & 1.11 & -0.11 & 1 & 0 \\
\hline 59. & 1 & 0.91 & 0.09 & 1.11 & -0.11 & 1 & 0 \\
\hline 60. & 1 & 0.909 & 0.091 & 1.11 & -0.11 & 0.993 & 0.007 \\
\hline 61. & 1 & 0.91 & 0.09 & 1.1 & -0.1 & 1 & 0 \\
\hline 62. & 1 & 0.904 & 0.096 & 1.1 & -0.1 & 1 & 0 \\
\hline 63. & 1 & 0.911 & 0.089 & 1.09 & -0.09 & 1 & 0 \\
\hline 64. & 1 & 0.907 & 0.093 & 1.09 & -0.09 & 1 & 0 \\
\hline 65. & 1 & 0.912 & 0.088 & 1.06 & -0.06 & 1 & 0 \\
\hline 66. & 1 & 0.909 & 0.091 & 1.05 & -0.05 & 1 & 0 \\
\hline 67. & 1 & 0.91 & 0.09 & 1.04 & -0.04 & 1 & 0 \\
\hline 68. & 1 & 0.907 & 0.093 & 1.04 & -0.04 & 1 & 0 \\
\hline 69. & 1 & 0.911 & 0.089 & 1.03 & -0.03 & 1 & 0 \\
\hline 70. & 1 & 0.907 & 0.093 & 1.01 & -0.01 & 1 & 0 \\
\hline 71. & 1 & 0.928 & 0.072 & 0.995 & 0.005 & 1 & 0 \\
\hline 72. & 1 & 0.909 & 0.091 & 0.99 & 0.01 & 1 & 0 \\
\hline 73. & 1 & 0.916 & 0.084 & 0.989 & 0.011 & 1 & 0 \\
\hline 74. & 1 & 0.904 & 0.096 & 0.983 & 0.017 & 1 & 0 \\
\hline 75. & 1 & 0.916 & 0.084 & 0.979 & 0.021 & 1 & 0 \\
\hline 76. & 1 & 0.912 & 0.088 & 0.965 & 0.035 & 1 & 0 \\
\hline 77. & 1 & 0.909 & 0.091 & 0.963 & 0.037 & 1 & 0 \\
\hline 78. & 1 & 0.909 & 0.091 & 0.959 & 0.041 & 1 & 0 \\
\hline 79. & 1 & 0.917 & 0.083 & 0.956 & 0.044 & 1 & 0 \\
\hline 80. & 1 & 0.905 & 0.095 & 0.956 & 0.044 & 1 & 0 \\
\hline 81. & 1 & 0.913 & 0.087 & 0.955 & 0.045 & 1 & 0 \\
\hline 82. & 1 & 0.901 & 0.099 & 0.954 & 0.046 & 1 & 0 \\
\hline 83. & 1 & 0.907 & 0.093 & 0.951 & 0.049 & 1 & 0 \\
\hline 84. & 1 & 0.909 & 0.091 & 0.918 & 0.082 & 1 & 0 \\
\hline 85. & 1 & 0.909 & 0.091 & 0.915 & 0.085 & 1 & 0 \\
\hline 86. & 1 & 0.909 & 0.091 & 0.903 & 0.097 & 1 & 0 \\
\hline 87. & 1 & 0.909 & 0.091 & 0.903 & 0.097 & 1 & 0 \\
\hline 88. & 1 & 0.909 & 0.091 & 0.896 & 0.104 & 1 & 0 \\
\hline 89. & 1 & 0.909 & 0.091 & 0.89 & 0.11 & 1 & 0 \\
\hline 90. & 1 & 0.907 & 0.093 & 0.884 & 0.116 & 1 & 0 \\
\hline 91. & 1 & 0.925 & 0.075 & 0.883 & 0.117 & 1 & 0 \\
\hline 92. & 1 & 0.914 & 0.086 & 0.882 & 0.118 & 0.925 & 0.075 \\
\hline 93. & 1 & 0.912 & 0.088 & 0.881 & 0.119 & 1 & 0 \\
\hline 94. & 1 & 0.905 & 0.095 & 0.88 & 0.12 & 0.856 & 0.144 \\
\hline 95. & 1 & 0.915 & 0.085 & 0.878 & 0.122 & 1 & 0 \\
\hline 96. & 1 & 0.2 & 0.8 & 0.872 & 0.128 & 1 & 0 \\
\hline 97. & 1 & 0.918 & 0.082 & 0.87 & 0.13 & 1 & 0 \\
\hline 98. & 1 & 0.921 & 0.079 & 0.864 & 0.136 & 1 & 0 \\
\hline 99. & 1 & 0.918 & 0.082 & 0.859 & 0.141 & 1 & 0 \\
\hline 100. & 1 & 0.921 & 0.079 & 0.85 & 0.15 & 1 & 0 \\
\hline
\end{tabular}

\section{Reference}

[1]. L. Aik and Y. Jayakumar, A study of neuro-fuzzy system in approximation-based problems, Matematika, 24(2), 2008, 113-130.

[2]. R. Belohlavek and G. Klir, Concepts and fuzzy logic (London, England, Massachusetts Institute of Technology, 2011).

[3]. Y. Chai, L. Jia and Z. Zhang, Mamdani model based adaptive neural fuzzy inference system and its application, World Academy of Science, Engineering and Technology, 51(2), 2009, 845-852.

[4]. E. Doğan, L. Saltabaş and E. Yıldırım (2007),"Adaptive neuro-fuzzy inference system application for estimating suspended sediment loads". International Earthquake Symposium Kocher, 5, 2007, 537-540.

[5]. R. Hndoosh, M. Saroa and S. Kumar, Fuzzy and adaptive neuro-fuzzy inference system of washing machine, European Journal of Scientific Research, 86(3), 2012, 443-459.

[6]. R. Hndoosh, M. Saroa and S. Kumar, A proposition for using mathematical models based on a fuzzy system with application, International Journal of Mathematical Sciences, 33(2), 2013, 1356-1374.

[7]. R. Hndoosh, M. Saroa and S. Kumar, Mathematical structure of fuzzy modeling of medical diagnoses by using clustering models, International Journal of Scientific \& Engineering Research, 5(8), 2014, 545-554

[8]. R. Hndoosh, M. Saroa and S. Kumar, Fuzzy mathematical model for detection of lung cancer using a multi-NFCLass with confusion fuzzy matrix for accuracy, International Journal of Mathematical and Computer Modeling, 19(1), $2014,1129-1141$.

[9]. C. Jose, R. Neil and W. Curt, Neural and adaptive systems (New York, John Wiley and SONS, Inc, 1999). 
[10]. G. Jandaghi, R. Tehrani, D. Hosseinpour, R. Gholipour and S. Shadkam, (2010), “Application of fuzzy-neural networks in multiahead forecast of stock price". African Journal of Business Management, 4, 2010, 903-914.

[11]. T. Kamel and M. Hassan, Adaptive neuro fuzzy inference system (ANFIS) for fault classification in the transmission lines, The Online Journal on Electronics and Electrical Engineering, 2, 2009, 164-169.

[12]. V. Marza and A. Seyyedi, Estimating development time of software projects using a neuro fuzzy approach, World Academy of Science, Engineering and Technology, 46, 2008, 575-579.

[13]. P. Nayak, K. Sudheer, D. Rangan and K. Ramasastri, A neuro-fuzzy computing technique for modeling hydrological time series, Journal of Hydrology, 291, 2004, 52-66.

[14]. A. Rameshkumar and S. Arumugam, A neuro-fuzzy integrated system for non-linear buck and quasi-resonant buck converter, European Journal of Scientific Research, 51(1), 2011, 66-78.

[15]. D. Ruan and P. Wang, Intelligent hybrid system: fuzzy logic, neural network and genetic algorithm (Kluwer Academic Publishers, 1997).

[16]. R. Rojas, Neural networks (Berlin, Springer-Verlag, 1996).

[17]. A. Samandar, A model of adaptive neural-based fuzzy inference system (ANFIS) for prediction of friction coefficient in open channel flow, Academic Journals Scientific Research and Essays, 6(5), 2011, 1020-1027.

[18]. J. Shing and R. Jang, ANFIS: Adaptive network-based fuzzy inference system, IEEE Transactions on Systems, Man, and Cybernetics, 23(3), 1993, 665-685.

[19]. M. P. Singh, M. K. Sharma and S. Kumar, Conjugate descent formulation of back propagation error in feed forward neural network, ORION: Journal of the Operations Research Society of South Africa, 25, 2009, 69-86.

[20]. S. Sivanandam and S. Deepa, Principal of soft computing (India, Wiley India (P) Ltd, 2008).

[21]. L. Wang, A course in fuzzy systems and control (Englewood Cliffs, NJ: Prentice-Hall International, Inc, 1997).

[22]. H. Sug, Improving the prediction accuracy of liver disoorder disease with oversampling, Applied Mathematics in Electrical and Computer Engineering, 7, 2012, 331-335.

[23]. A. Gulia, R. Vohra, P. Rani, Liver patient classification using intelligent techniques, International Journal of Computer Science and Information Technologies, 5(4), 2014, 5110-5115.

[24]. H. Sug, Performance comparison of different over-sampling rates of decision trees for the class of higher error rate in the liver data set, International Journal of Mathematics and Computers in Simulation, 6(2), 2012, 282-289. 